MPI-PhT/94-87

Submitted to

December 9, 1994

Nucl. Phys. B

gr-qc/9412039

\title{
Gravitating Monopole Solutions II
}

\author{
Peter Breitenlohner, Peter Forgács 凹, and Dieter Maison \\ Max-Planck-Institut für Physik \\ - Werner Heisenberg Institut - \\ Föhringer Ring 6 \\ 80805 Munich (Fed. Rep. Germany)
}

\begin{abstract}
We present analytical and numerical results for static, spherically symmetric solutions of the Einstein-Yang-Mills-Higgs equations corresponding to magnetic monopoles and non-abelian magnetically charged black holes. In the limit of infinite Higgs mass we give an existence proof for these solutions. The stability of the abelian extremal Reissner-Nordstrøm black holes is reanalyzed.
\end{abstract}

\section{Introduction}

In the present paper we continue our investigation of magnetically charged nonabelian black holes, resp. gravitating magnetic monopoles, started in [1], referred to as I. The latter are solutions of an Einstein-Yang-Mills-Higgs (EYMH) theory with the Higgs field in the adjoint representation. In the following we restrict ourselves to an $S U(2)$ gauge theory as in I.

Gravitating magnetic monopoles were first considered in 22 and later studied numerically together with black holes in some detail in [1, 3, 4]. The numerical studies revealed a number of interesting phenomena for both of the cases when the strength of the gravitational force is either very weak or becomes comparable to that of the YM interaction. The relevant dimensionless parameter characterizing the relative strength of the gravitational interaction is the mass ratio $\alpha=M_{\mathrm{W}} / g M_{\text {Planck }}$ where $M_{\text {Planck }}=1 / \sqrt{G}$ is the the Planck mass and $M_{\mathrm{W}}$ is the mass of the YM field (due to the Higgs effect). It has been found that regular monopoles exist only for $\alpha \leq \alpha_{\max }$ of order one. The precise value of $\alpha_{\max }$ depends on the second dimensionless mass ratio $\beta=M_{\mathrm{H}} / M_{\mathrm{W}}$ where $M_{\mathrm{H}}$ is the mass of the Higgs field. In addition to the gravitating monopole solution tending to its flat limit for $\alpha \rightarrow 0$ a discrete family of radially excited monopole solutions was found for arbitrarily small $\alpha$ (and $\alpha<\sqrt{3} / 2$ ) [1] having no counterpart in flat space.

Another new feature without analogue in flat space is the existence of nonabelian magnetically charged black holes. They were found to exist in a certain bounded domain in the $\left(\alpha, r_{h}\right)$ plane where $r_{h}$ denotes the radius of the black hole horizon. The precise form of this domain again depends on $\beta$.

\footnotetext{
${ }^{1}$ Permanent address: Research Institute for Particle and Nuclear Physics H-1525 Budapest 114, P.O.Box 49
} 
Whereas the emphasis in I was mainly on numerical results, the present work is devoted more to to analytical considerations and a qualitative understanding of the numerical results.

It would be clearly desirable to give analytical proofs for the existence of the monopole solutions found by numerical integration as it was achieved [5, 6] for the discrete family of solutions of the EYM theory found by Bartnik and McKinnon [f] and the related black holes [8]. One of the main results of this paper is an existence proof of magnetically charged non abelian black holes, resp. magnetic monopoles in the limiting case $\beta \rightarrow \infty$ when the Higgs field is frozen to its vacuum expectation value. In this case the analysis of the problem is considerably simplified while many features of the full problem (i.e., the one with a dynamical Higgs field) do carry over. Although the corresponding spherically symmetric ansatz for the Higgs field is singular at the origin, there exist still 'quasi regular' solutions with finite mass. The corresponding gravitational field develops a 'conical singularity' at the origin, whereas the YM field is still differentiable. The angular deficit leading to this conical singularity is the same as the one found for 'global monopoles' [9]. The only difference is that in the latter case it has been transferred to large values of $r$ using $1 / M_{\mathrm{H}}$ as length scale instead of $1 / M_{\mathrm{W}}$ used here.

The maximal value of the mass $\alpha$ (measured in units of the Planck mass) for which the quasi regular solution exists was found to be $1 / \sqrt{2}$ [何. This is exactly the value where the angular deficit becomes $4 \pi$, i.e., the conical singularity changes its nature. The behaviour of the solution at the origin is then rather different from the one for smaller values of $\alpha$. A similar problem arises, when one considers black holes with a degenerate horizon. In fact, the quasi regular solution emerges in the limit $r_{h} \rightarrow 0$ of such extremal black holes. Even for local solutions the proof of existence for extremal black holes (and their numerical construction) requires certain modifications of the methods used in I. These will be described in Sect. \#, after discussing the field equations in Sect. 2 and the necessary boundary conditions in Sect. 3

Sect. 5 is devoted to an analysis of the global behaviour of all solutions with the boundary conditions at $r=0$, resp. $r_{h}$ discussed in Sect. 3. Methods and results of this analysis, leading to a complete classification, follow closely those of [6]. As a consequence of this classification the existence of global black hole solutions for the massive EYM theory $(\beta=\infty)$ is demonstrated for a certain domain of the $\left(\alpha, r_{h}\right)$ plane. Analyzing the limiting behaviour of the solutions when the horizon degenerates, resp. $r_{h}$ tends to zero the existence proof can be extended to extremal black holes, resp. quasi regular solutions.

In Sect. 6 we discuss the problems involved in the numerical solution of the relevant boundary value problem with particular emphasis on the difficulties arising from the singular nature of the boundary points. Furthermore we try to give a qualitative understanding of the various mechanisms delimiting the existence region of global (regular and black hole) solutions as a function of the parameters $\alpha$ and $\beta$.

Some numerical solutions are also presented to illustrate the usefulness of the techniques described in Sect. $⿴$ in a case, which could not be dealt with previously $(\beta=\infty, \alpha=1 / \sqrt{2})$.

In the last section we address the stability of the extremal Reissner-Nordstrøm solution. We demonstrate that it becomes unstable (independently of $\beta$ ) for precisely $\alpha=\sqrt{3} / 2$ and not for $\alpha=1$ as it has been claimed previously [4, 10]. This result complies well with the numerically observed bifurcation of the nonabelian black holes of radius $r_{h}=\alpha$ with the extremal RN solution for all values of $\beta$.

\section{Ansatz and Field Equations}

We are interested in static, spherically symmetric solutions of the EYMH equations. In this case the metric tensor of the space-time can be parametrized as 12

$$
d s^{2}=e^{2 \nu(R)} d t^{2}-e^{2 \lambda(R)} d R^{2}-r^{2}(R) d \Omega^{2},
$$


where $d \Omega^{2}=d \theta^{2}+\sin ^{2} \theta d \varphi^{2}$. Whereas the functions $e^{\nu}$, resp. $4 \pi r^{2}$ have a geometrical significance as the length of the time-translation Killing vector, resp. the surface area of the invariant 2spheres the function $e^{\lambda}$ is gauge dependent, i.e., depends on the choice of the radial coordinate $R$. A very convenient choice is obtained putting $e^{\lambda}=r / R$ corresponding to isotropic coordinates for the 3 -spaces $t=$ const.

For the $S U(2)$ Yang-Mills field $W_{\mu}^{a}$ we use the standard minimal spherically symmetric (purely 'magnetic') ansatz

$$
W_{\mu}^{a} T_{a} d x^{\mu}=W(R)\left(T_{1} d \theta+T_{2} \sin \theta d \varphi\right)+T_{3} \cos \theta d \varphi,
$$

where $T_{a}$ denote the generators of $S U(2)$ and for the Higgs field we assume the form

$$
\Phi^{a}=H(R) n^{a}
$$

where $n^{a}$ is the unit vector in the radial direction. The reduced EYMH action can be expressed as

$$
S=-\int d \operatorname{Re}^{(\nu+\lambda)}\left[\frac{1}{2}\left(1+e^{-2 \lambda}\left(\left(r^{\prime}\right)^{2}+\nu^{\prime}\left(r^{2}\right)^{\prime}\right)-e^{-2 \lambda} r^{2} V_{1}-V_{2}\right]\right.
$$

with

$$
V_{1}=\frac{\left(W^{\prime}\right)^{2}}{r^{2}}+\frac{1}{2}\left(H^{\prime}\right)^{2}
$$

and

$$
V_{2}=\frac{\left(1-W^{2}\right)^{2}}{2 r^{2}}+\frac{\beta^{2} r^{2}}{8}\left(H^{2}-\alpha^{2}\right)^{2}+W^{2} H^{2} .
$$

Through a suitable rescaling we have achieved that the action depends only on the dimensionless parameters $\alpha$ and $\beta$ representing the mass ratios $\alpha=M_{W} \sqrt{G} / g=M_{W} / g M_{\mathrm{Pl}}$ and $\beta=M_{H} / M_{W}$ ( $g$ denoting the gauge coupling).

In order to derive the gravitational field equations we have to employ independent variations of the functions $\nu, \lambda$ and $r$, inserting the gauge choice $e^{\lambda}=r / R$ afterwards.

The resulting equations are

$$
\begin{aligned}
\frac{1}{2}\left(1-\left(\frac{R r^{\prime}}{r}\right)^{2}-2 R^{2} \nu^{\prime} \frac{r^{\prime}}{r}\right)+R^{2} V_{1}-V_{2} & =0, \\
\frac{1}{2}\left(1-\left(\frac{R r^{\prime}}{r}\right)^{2}-2 \frac{R}{r}\left(R r^{\prime}\right)^{\prime}\right)-R^{2} V_{1}-V_{2} & =0, \\
R\left(\frac{R r^{\prime}}{r}\right)^{\prime}+R e^{-\nu}\left(R e^{\nu} \nu^{\prime}\right)^{\prime}+R^{2} r \frac{\partial V_{1}}{\partial r}+r \frac{\partial V_{2}}{\partial r} & =0, \\
R\left(\frac{R}{r} e^{\nu} W^{\prime}\right)^{\prime}-e^{\nu} r W\left(\frac{W^{2}-1}{r^{2}}+H^{2}\right) & =0, \\
R\left(R r e^{\nu} H^{\prime}\right)^{\prime}-e^{\nu} r H\left(2 W+\frac{\beta^{2} r^{2}}{2}\left(H^{2}-\alpha^{2}\right)\right) & =0 .
\end{aligned}
$$

In order to obtain an autonomous first order system we introduce the coordinate $\tau=\ln R$ (with $\cdot=d / d \tau)$ and new variables for the first derivatives through

$$
N \equiv \frac{\dot{r}}{r}, \quad \kappa \equiv \dot{\nu}+N, \quad U \equiv \frac{\dot{W}}{r}, \quad \text { and } \quad V \equiv \dot{H} .
$$

Thus we obtain the system of equations

$$
\begin{aligned}
\dot{r} & =r N \\
\dot{N} & =(\kappa-N) N-2 U^{2}-V^{2}, \\
\dot{\kappa} & =1-\kappa^{2}+2 U^{2}-\frac{\beta^{2} r^{2}}{2}\left(H^{2}-\alpha^{2}\right)^{2}-2 H^{2} W^{2},
\end{aligned}
$$




$$
\begin{aligned}
\dot{W} & =r U, \\
\dot{U} & =\frac{W\left(W^{2}-1\right)}{r}+r H^{2} W-(\kappa-N) U, \\
\dot{H} & =V, \\
\dot{V} & =\frac{\beta^{2} r^{2}}{2}\left(H^{2}-\alpha^{2}\right) H+2 W^{2} H-\kappa V,
\end{aligned}
$$

together with the constraint

$$
2 \kappa N=1+N^{2}+2 U^{2}+V^{2}-2 V_{2},
$$

derived from Eq. (7a).

A certain simplification occurs taking the formal limit $\beta \rightarrow \infty$ of infinite Higgs mass. In this limit the Higgs field is frozen at its 'vacuum' value $H \equiv \alpha$ and at the same time the last two of the field equations are discarded. As seen from the ansatz (3) the Higgs field is singular at $r=0$, but this singularity is not directly visible in the radial field equations

$$
\begin{aligned}
\dot{r} & =r N, \\
\dot{N} & =(\kappa-N) N-2 U^{2}, \\
\dot{\kappa} & =1-\kappa^{2}+2 U^{2}-2 \alpha^{2} W^{2}, \\
\dot{W} & =r U, \\
\dot{U} & =\frac{W\left(W^{2}-1\right)}{r}+r \alpha^{2} W-(\kappa-N) U,
\end{aligned}
$$

and the constraint

$$
2 \kappa N=1+N^{2}+2 U^{2}-\frac{\left(1-W^{2}\right)^{2}}{r^{2}}-2 \alpha^{2} W^{2} .
$$

These equations may be interpreted as describing a massive YM field (of mass $\alpha$ ). For $\alpha=0$ the equations coincide with the Eqs. $(49,50)$ of [6].

Special solutions of Eqs. (9), resp. (11) are the abelian Reissner-Nordstrøm (RN) black holes with $\left(r_{h}\right.$ denoting the radius of the horizon)

$$
W \equiv 0, \quad H \equiv \alpha, \quad N^{2}=1-\frac{1+r_{h}^{2}}{r r_{h}}+\frac{1}{r^{2}}
$$

Expressing $r$ as a function of $\tau$ yields

$$
r=r_{h} \cosh ^{2} \frac{\tau}{2}-\frac{1}{r_{h}} \sinh ^{2} \frac{\tau}{2}, \quad N=\frac{\left(r_{h}^{2}-1\right) \sinh \tau}{2 r r_{h}}, \quad \kappa=\operatorname{coth} \tau,
$$

for the regular RN solution with $r_{h}>1$ and

$$
r=1+e^{\tau}, \quad N=\frac{1}{1+e^{-\tau}}, \quad \kappa \equiv 1,
$$

for the extremal RN solution with $r_{h}=1$.

\section{Boundary conditions}

The field Eqs. (9) have to be complemented with suitable boundary conditions. For physical reasons we are only interested in solutions with a regular origin $r=0$ or black holes. In both cases the corresponding boundary points are singular points of the field equations. Whereas for $r=0$ this is obvious from Eqs. (9) it is less obvious in the case of black holes. Using Eqs. (9a) 
and $e^{\lambda}=r / R$ one obtains the relation $N=e^{-\lambda} d r / d R$. Since $e^{-\lambda}$ has to vanish on the horizon of the black hole also $N$ vanishes there. This, however, leads to a divergence of $\kappa$ in view of the constraint Eq. (10).

For asymptotically flat solutions we also have to impose boundary conditions at $r=\infty$, another singular point of the equations.

Usually the regular solutions at some singular point are determined by a number of free parameters strictly smaller than that for a non-singular point. A standard procedure to determine these free parameters is to expand the solution in a formal Taylor series in the independent variable and read off the undetermined coefficients. If this Taylor series converges it defines a family of local solutions parametrized directly at the singular point. There are, however, cases where this method fails, e.g., for black holes with a degenerate horizon. Yet, there is a more general method to obtain local solutions at singular (fixed) points of 'dynamical systems' (i.e., systems of first order ODE's). One tries to find a linearization of the differential operator which allows to characterize the behaviour of solutions near the singular point. In the case of a hyperbolic fixed point regular solutions of the linearized system lie on a linear subspace ('stable manifold'). Regular solutions of the full non-linear system lie on a corresponding curved stable manifold of the fixed point, whose existence is guaranteed by standard textbook theorems (e.g., [13, 14]). The method to determine the stable manifold through a system of integral equations solvable by iteration yields a viable numerical method to obtain local solutions even in cases, where the Taylor series approach fails.

After these general remarks we turn to a detailed discussion of the various boundary conditions. The behaviour of regular solutions at $r=0$ was already treated in I, although with the coordinate choice $r=R$ (Schwarzschild coordinates). One finds the asymptotic behaviour

$$
\begin{aligned}
& W(r)=1-b r^{2}+O\left(r^{4}\right), \\
& H(r)=a r+O\left(r^{3}\right), \\
& N(r)=1-\frac{1}{2}\left(a^{2}+4 b^{2}+\frac{\beta^{2} \alpha^{4}}{12}\right) r^{2}+O\left(r^{4}\right),
\end{aligned}
$$

where $a$ and $b$ are free parameters. In view of the preceding discussion we interprete the expansion of $W$ etc. in terms of $r$ as an approximation to the 'stable manifold', i.e., as a relation between dependent variables. In this case the parameters $a$ and $b$ are defined as the limits of $H / r$ and $\left(1-W^{2}\right) / r^{2}$ for $\tau \rightarrow-\infty$, i.e., at the singular point.

Next we turn to the case $\beta=\infty$ with the field Eqs. (9). Also this case has already been discussed in I, where we found

$$
\begin{aligned}
W(r) & =1-\frac{r^{2}}{4}+\ldots+b r^{\gamma}+o\left(r^{\gamma}\right), \\
N(r) & =\sqrt{1-2 \alpha^{2}}\left(1+\frac{1}{24} \frac{8 \alpha^{2}-3}{1-2 \alpha^{2}} r^{2}+\ldots+b r^{\gamma}+o\left(r^{\gamma}\right)\right), \\
\gamma & =\frac{1}{2}\left(1+\sqrt{\frac{9-2 \alpha^{2}}{1-2 \alpha^{2}}}\right),
\end{aligned}
$$

the dots representing even polynomials in $r$ of degree smaller than $\gamma$. As long as $\alpha^{2}<1 / 2$ the stable manifold can still be parametrized by $b$, although to define it through the limit of $\left(1-W^{2}\right) / r^{\gamma}$ one has first to substract a polynomial of degree smaller than $\gamma$. We remark that whenever $\gamma$ is an even integer larger than 2, i.e., $\alpha=\sqrt{\frac{1}{2}-1 / 2 m(2 m-1)}$ with $m=2,3, \ldots$ logarithms appear in Eqs. (17).

The most important new feature is the behaviour of $N$ at $r=0$. Whereas $N(0)=1$ for all finite $\beta$, now $N(0)=\sqrt{1-2 \alpha^{2}}$ implying an angular deficit and a curvature singularity at the 

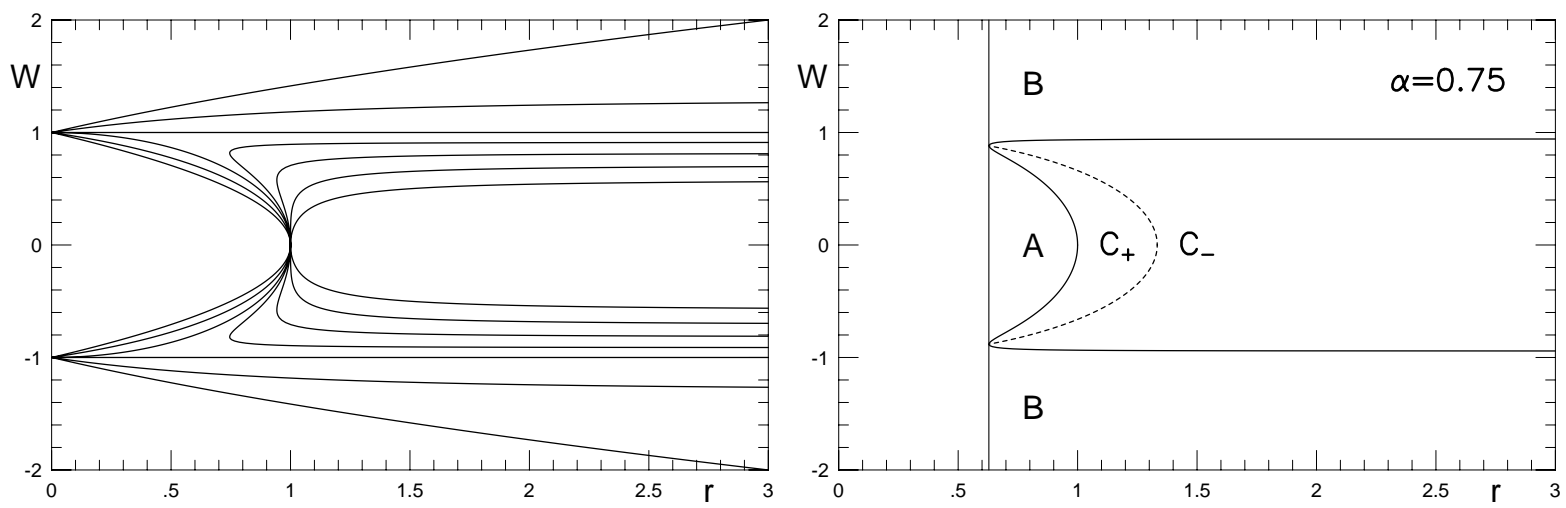

Figure 1: The curve $N_{1}=0$ for $\beta=\infty$ and $\alpha^{2}=0,0.3,0.5,0.6,0.75,1$, and 1.5

Figure 2: Some regions of the $(r, W)$ plane for $\alpha=0.75$ and $\beta=\infty$

origin. It is induced by the singularity of the Higgs field at $r=0$. The asymptotic expression for $N(r)$ shows that $\alpha$ has to be restricted to $0 \leq \alpha<1 / \sqrt{2}$. In the limit $\alpha \rightarrow 1 / \sqrt{2}$ the expression for $\gamma$ and the conditions (17) become meaningless. This is a case where the 'stable manifold' itself is well-defined, however, its parametrization at the singular point breaks down. A precise formulation of this case will be given in the next section.

The boundary conditions for regular black holes at the horizon were already given in I in Schwarzschild coordinates. For later convenience we repeat them here using the coordinate $\tau$. As already mentioned the function $\kappa(\tau)$ is singular at the horizon (which we choose to put at $\tau=0$ ), but $\kappa(\tau)-1 / \tau$ turns out to be regular. One obtains

$$
\begin{aligned}
& r(\tau)=r_{h}\left(1+N_{1} \frac{\tau^{2}}{2}\right)+O\left(\tau^{4}\right) \\
& N(\tau)=N_{1}\left(\tau+O\left(\tau^{3}\right)\right)-\left(W_{1}^{2}+\frac{1}{2} H_{1}^{2}\right) \tau^{3}+O\left(\tau^{5}\right) \\
& W(\tau)=W_{h}+r_{h} W_{1} \frac{\tau^{2}}{2}+O\left(\tau^{4}\right) \\
& H(\tau)=H_{h}+H_{1} \frac{\tau^{2}}{2}+O\left(\tau^{4}\right)
\end{aligned}
$$

where

$$
N_{1}=\frac{1}{2}-\left.V_{2}\right|_{h}, \quad W_{1}=\left.\frac{r_{h}}{4} \frac{\partial V_{2}}{\partial W}\right|_{h}, \quad H_{1}=\left.\frac{1}{2} \frac{\partial V_{2}}{\partial H}\right|_{h},
$$

and $r_{h}, W_{h}$, and $H_{h}$ are undetermined parameters. For $\beta=\infty$ one has to substitute $H \equiv \alpha$, $H_{1} \equiv 0$ and omit Eq. (18d). In order to have a regular horizon we have to require $N_{1}$ to be positive. For given $r_{h}, \alpha, \beta$ the inequality $N_{1}>0$ determines some bounded (allowed) region in the $\left(W_{h}, H_{h}\right)$ plane. In the limiting case $\beta=\infty$ the expansions simplify in an obvious way and the allowed region of the $\left(r_{h}, W_{h}\right)$ plane is bounded by the quartic curve $N_{1}=0$. In Fig. 1 these quartic curves are shown for various values of $\alpha$, the allowed region is shown as $C=C_{+} \cup C_{-}$ for $\alpha=0.75$ in Fig. 2 .

If $\beta>2$ the regions in the $\left(W_{h}, H_{h}\right)$ plane allowed by the condition $N_{1} \geq 0$ shrink to points for certain values of $\alpha$ and $r_{h}$. In this case $W_{h}$ and $H_{h}$ are fixed by the conditions

$$
\begin{array}{r}
\frac{\left(W_{h}^{2}-1\right)^{2}}{r_{h}^{2}}+2 W_{h}^{2} H_{h}^{2}+\frac{\beta^{2}}{4} r_{h}^{2}\left(H_{h}^{2}-\alpha^{2}\right)^{2}=1, \\
W_{h}^{2}-1+r_{h}^{2} H_{h}^{2}=0,
\end{array}
$$




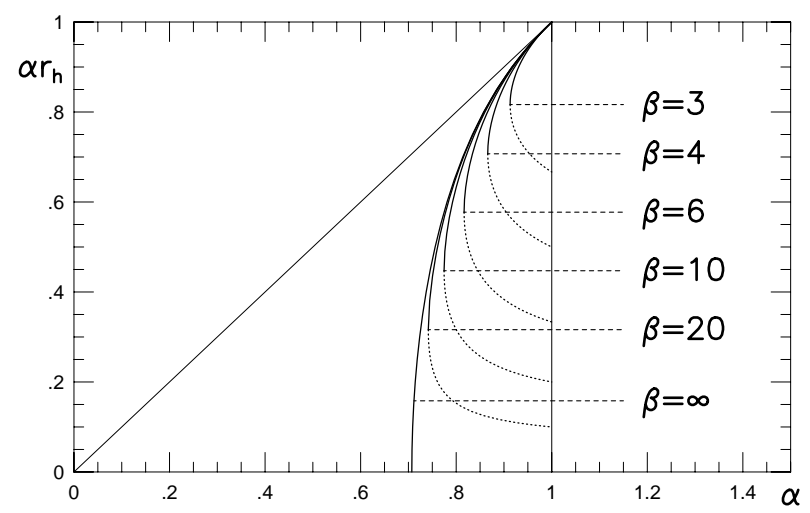

Figure 3: The curves $\alpha_{e} r_{h}$ vs. $\alpha_{e}$ for $\beta=3,4,6,10,20$, and $\infty$

$$
\frac{\beta^{2}}{4} r_{h}^{2}\left(H_{h}^{2}-\alpha^{2}\right)+W_{h}^{2}=0
$$

Nevertheless there still exists a two parameter family of local solutions, but members of this family cannot be characterized by their behaviour at the horizon. The horizon is degenerate since Eqs. (20) imply $N_{1}=W_{1}=H_{1}=0$.

It is convenient to give the solution of Eqs. (20) in parametric form:

$$
\begin{aligned}
r_{h}^{2} H_{h}^{2} & =1-W_{h}^{2}, \\
\alpha^{2} r_{h}^{2} & =1-\left(1-\frac{4}{\beta^{2}}\right) W_{h}^{2}, \\
r_{h}^{2} & =1-\left(1-\frac{4}{\beta^{2}}\right) W_{h}^{4} .
\end{aligned}
$$

When $W_{h}$ increases from 0 to 1 for some fixed $\beta>2$, then $H_{h}$ decreases from 1 to $0, r_{h}$ decreases from 1 to $2 / \beta$, and $\alpha$ first decreases from 1 to a minimal value and then increases to 1 ; the minimal value $\alpha_{\min }=\sqrt{(\beta+2) / 2 \beta}$ is reached for $W_{h}=\sqrt{\beta /(\beta+2)}$ where $r_{h}=2 / \sqrt{\beta+2}$ and $H_{h}=1 / \sqrt{2}$. Solving Eqs. (21) for $\alpha$ in terms of $r_{h}$ one obtains a function $\alpha_{e}\left(r_{h}\right)$ given by

$$
\alpha_{e}^{2}=\frac{1-\sqrt{\left(1-4 / \beta^{2}\right)\left(1-r_{h}^{2}\right)}}{r_{h}^{2}} .
$$

This function is shown in Fig. 3 for various values of $\beta$.

The conditions $N_{1}=W_{1}=H_{1}=0$ for a degenerate horizon have some more solutions besides Eqs. (20), discussed in more detail in Sect. 1 .

Finally let us consider the b.c. when $r \rightarrow \infty$. Imposing

$$
N(r)=1-\frac{M}{r}+O\left(\frac{1}{r^{2}}\right)
$$

ensures asymptotic flatness. Then from the field eqs. one easily finds:

$$
\begin{aligned}
& W(r)=C e^{-\alpha \sigma}\left(1+O\left(\frac{1}{r}\right)\right), \\
& H(r)=\alpha-B \frac{e^{-\alpha \beta \sigma}}{r}\left(1+O\left(\frac{1}{r}\right)\right), \text { for } \beta<2, \\
& H(r)=\alpha-\frac{2 C^{2}}{\alpha\left(\beta^{2}-4\right)} \frac{e^{-2 \alpha \sigma}}{r^{2}}\left(1+O\left(\frac{1}{r}\right)\right), \text { for } \beta>2,
\end{aligned}
$$


where $\sigma=r+M \ln r$ and $M, B$, and $C$ are free parameters, i.e., there exists a three-parameter family of asymptotically flat solutions. The asymptotic behaviour of the Higgs field $H(r)$ depends crucially on $\beta$. When $\beta \geq 2$ its asymptotic decay is dominated by the non-linear terms in Eqs. (9g) and it is not possible to fully parametrize the stable manifold at the singular point $r=\infty$.

\section{Local Existence}

In the preceding section we have analyzed the behaviour of solutions of Eqs. (9, 10) or (11, 12) that remain regular at one of the singular points $(r=0, r=\infty$, or a horizon). We have found that there are families of such solutions depending on one or several free parameters. The arguments were, however, based on a formal power series expansion. In order to show that such solutions exist as real analytic functions we apply standard techniques to linearize non-linear systems at a singular point.

For regular black holes $\kappa$ has a pole at the horizon for finite $\tau$ and in Eqs. (18) we have chosen $\tau=0$ at the horizon. In all other cases discussed in Sect. 3 the dependent variables have a limit as $\tau \rightarrow \pm \infty$ such that all the right hand sides of Eqs. (9), resp. (11) vanish. The behaviour of solutions near such a singular or fixed point can be characterized by a 'stable manifold' (see, e.g., 13 p.330ff). Consider a system of autonomous first order differential eqs. for $m+n$ functions $y=\left(u_{1}, \ldots, u_{m}, v_{1}, \ldots, v_{n}\right)$

$$
\frac{d u}{d t}=A u+f(y), \quad \frac{d v}{d t}=-B v+g(y),
$$

where all eigenvalues $\mu$, resp. $\lambda$ of the constant matrices $A$, resp. $B$ have a positive real part, and $f(y), g(y)$ are $O\left(\|y\|^{2}\right)$ and analytic for $\|y\|$ sufficiently small. There exists an $n$-dimensional stable manifold (for $t \rightarrow+\infty$ ) of initial data $y(0)=(\psi(v), v)$ such that $y \rightarrow 0$ as $t \rightarrow+\infty$. Identifying solutions with the same orbit (only differing by a shift in $t$ ) there exists an $(n-1)$ parameter family of such equivalence classes of solutions on the stable manifold. The functions $\psi_{i}(v)$ are $O\left(\|v\|^{2}\right)$ and analytic for $\|v\|$ sufficiently small. For such $v$ and $t \geq 0$ the solutions are analytic in $v$ and $t$. Due to the presence of non-linear terms this result does not, however, imply that $e^{B t} v(t)$ has a limit for $t \rightarrow \infty$. For solutions off the stable manifold the distance $\|u-\psi(v)\|$ increases exponentially as long as $\|y\|$ is small.

Similarly there exists an $m$-dimensional stable manifold (for $t \rightarrow-\infty$ ) of initial data $y(0)=$ $(u, \varphi(u))$ such that $y \rightarrow 0$ as $t \rightarrow-\infty$.

It is sometimes possible to characterize solutions on the stable manifold by their behaviour

at the singular point. In [1] we have derived the following result for these cases (see also 114 p.304ff): Consider a system of first order differential eqs. for $m+n$ functions $y=(u, v)$

$$
\begin{aligned}
& s \frac{d u_{i}}{d s}=s f_{i}(s, y), \quad i=1, \ldots, m, \\
& s \frac{d v_{i}}{d s}=-\lambda_{i} v_{i}+s g_{i}(s, y), \quad i=1, \ldots, n,
\end{aligned}
$$

with constants $\lambda_{i}>0$ and let $\mathcal{C}$ be an open subset of $R^{m}$ such that $f_{i}, g_{i}$ are analytic in a neighbourhood of $s=0, y=(c, 0)$ for all $c \in \mathcal{C}$. There exists an $m$-parameter family of local solutions $y_{c}(s)$ analytic in $c$ and $s$ for $c \in \mathcal{C},|s|<s_{0}(c)$ such that $y_{c}(0)=(c, 0)$.

We have used this result in [1] to show that there exists a two-parameter family of solutions of Eqs. (9.10) with regular origin where $W, H$, and $N$ are analytic in $r, a$, and $b$ and satisfy the boundary conditions (16). Finally $r$ is an analytic function of $\tau$ due to Eq. (9a) and $\kappa$ as computed from the constraint (10) is analytic as well. 
Let us next consider the 'quasi regular' solutions of Eqs. (11, 12) with a conical singularity at the origin. Assuming $\alpha<1 / \sqrt{2}$ we define $\nu=\sqrt{1-2 \alpha^{2}}$, introduce

$$
\bar{W}=\frac{(W-1)}{r}-\frac{r}{4}, \quad \bar{U}=\frac{U}{\nu}+\frac{r}{2}, \quad \bar{N}=N-\nu, \quad \bar{\kappa}=\kappa-\nu,
$$

and use the constraint (12) to express $\bar{\kappa}$ in terms of $r, \bar{W}, \bar{U}$, and $\bar{N}$. Taking the linear combinations $X_{+}=(\gamma-1) \bar{W}+\bar{U}$ and $X_{-}=\gamma \bar{W}-\bar{U}$ with $\gamma$ from Eq. (17]c) we obtain a system of equations in the form (25) for $t=\tau, u=\left(r, X_{+}\right), v=\left(\bar{N}, X_{-}\right)$with eigenvalues $\mu=(\nu,(\gamma-1) \nu), \lambda=(\nu, \gamma \nu)$. Hence there exists a two-dimensional stable manifold for $\tau \rightarrow$ $-\infty$, i.e., a one-parameter family of quasi regular solutions that can be characterized, e.g., by $X_{+}(r)=-b(r) r^{(\gamma-1)}$. As long as $\gamma<4$ the system can be written in the form (26), and hence $b(r)$ has a limit for $r \rightarrow 0$; for larger $\gamma$ higher powers of $r$ must be subtracted from $X_{ \pm}$in order to parametrize the solutions at the singular point $r=0$ (compare Eq. (17a)).

To show the existence of solutions satisfying the black hole boundary conditions (18) we observe that Eqs. (9) can be written in the form (26) with $s=\tau, u=\left(r, W, H, 2 \kappa N-2 U^{2}-V^{2}\right)$, $v=\left(U, V, \kappa-\frac{1}{\tau}\right)$, and $\lambda=(1,1,2)$. One free parameter is fixed by the constraint (10), hence there exists a three-parameter family of local solutions analytic in $r_{h}, W_{h}, H_{h}$, and $\tau$. These solutions describe a regular horizon as long as $\left(r_{h}, W_{h}, H_{h}\right)$ are chosen such that $N_{1}>0$. Since $W, H$, and $N^{2}$ depend only on $\tau^{2}$ and $N_{1} \neq 0$ they may be expressed as analytic funtions of $r$. Similarly there exists a two-parameter family of local solutions of Eqs. (11, 12) analytic in $r_{h}$, $W_{h}$, and $\tau$ (or $r$ ).

A degenerate horizon occurs if $(r, N, \kappa, W, U, H, V)$ tend to a fixed point of Eqs. (9) with $N=U=V=0$ as $\tau \rightarrow-\infty$. For such a fixed point $N_{1}, W_{1}$, and $H_{1}$ in Eq. (19) have to vanish, furthermore $\kappa^{2}=-r \partial V_{2} / \partial r$ requiring $r \partial V_{2} / \partial r \leq 0$. (For $r \partial V_{2} / \partial r>0$ there are infinitely many zeros and poles of $\kappa$ instead of a fixed point.) There are four possible types of fixed points:

1. $r_{h}=1, W_{h}=0$, and $H_{h}=\alpha$,

2. solutions of Eqs. (20),

3. $r_{h}=2 / \alpha^{2} \beta, W_{h}=1$, and $H_{h}=0$,

4. $1-r_{h}^{2}+\alpha^{4} \beta^{2} r_{h}^{4} / 4=0, W_{h}=0$, and $H_{h}=0$.

In order to analyze the behaviour near the fixed points we introduce $\bar{r}=r-r_{h}, \bar{\kappa}=\kappa-\kappa_{h}$, $\bar{W}=W-W_{h}$, and $\bar{H}=H-H_{h}$. Using the constraint (10) to express $\bar{r}$ in terms of the other quantities we obtain $\bar{r}=N r_{h} / \kappa_{h}$ up to non-linear terms. The resulting linearized equations have the form $\dot{y}=M y$ with $y^{T}=(N, \bar{W}, U, \bar{H}, V, \bar{\kappa})$ and

$$
M=\left(\begin{array}{cccccc}
\kappa_{h} & 0 & 0 & 0 & 0 & 0 \\
0 & 0 & r_{h} & 0 & 0 & 0 \\
\cdot & \frac{3 W_{h}^{2}-1}{r_{h}}+r_{h} H_{h}^{2} & -\kappa_{h} & 2 r_{h} W_{h} H_{h} & 0 & 0 \\
0 & 0 & 0 & 0 & 1 & 0 \\
\cdot & 4 W_{h} H_{h} & 0 & \beta^{2} r_{h}^{2} \frac{3 H_{h}^{2}-\alpha^{2}}{2}+2 W_{h}^{2} & -\kappa_{h} & 0 \\
\cdot & \cdot & 0 & 0 & -2 \kappa_{h}
\end{array}\right),
$$

where $\cdot$ denotes some non-vanishing matrix elements whose precise form is not required. The matrix $M$ has a positive eigenvalue $\kappa_{h}$ and a negative one $-2 \kappa_{h}$. In order to determine the remaining four eigenvalues $\gamma_{i}$ let us study each of the possible fixed points in some detail.

First there is, for all $\alpha$ and $\beta$, the fixed point $r=\kappa=1, W=0, H=\alpha$ with eigenvalues

$$
\begin{aligned}
& \gamma_{1,2}=-\frac{1}{2} \pm i \omega \quad \text { with } \quad \omega=\sqrt{\frac{3}{4}-\alpha^{2}}, \\
& \gamma_{3,4}=-\frac{1}{2} \pm \sqrt{\frac{1}{4}+\alpha^{2} \beta^{2}} .
\end{aligned}
$$


The two eigenvalues $\gamma_{3,4}$ due to the Higgs field are real and have opposite sign for $\alpha \beta \neq 0$. The two eigenvalues $\gamma_{1,2}$ due to the Yang-Mills field are complex conjugate and have a negative real part for $\alpha<\sqrt{3} / 2$, are both negative for $\sqrt{3} / 2<\alpha<1$, and have opposite sign for $\alpha>1$.

For $\alpha>1$ there exists a two-parameter family of local solutions with a degenerate horizon approaching the fixed point as $\tau \rightarrow-\infty$. For $\alpha<1$ there is a one-parameter family with $W \equiv 0$. The extremal RN solution with $W \equiv 0$ and $H \equiv \alpha$ is a member of these families. In addition for $\alpha>1$, resp. $\alpha<1$ there exists a two-, resp. three-parameter family of local solutions approaching the fixed point as $\tau \rightarrow+\infty$. For $\alpha<\sqrt{3} / 2$ the asymptotic behaviour of $W$ and $U$ is dominated by the linearized equations, i.e.,

$$
W \approx C e^{-\tau / 2} \sin (\omega \tau+\theta)
$$

whereas $W$ monotonously decreases for $\alpha \geq \sqrt{3} / 2$ and $\tau$ sufficiently large. The two-parameter family of solutions for $\alpha>1$ is characterized by $e^{\tau / 2} W \rightarrow 0$ as $\tau \rightarrow+\infty$, whereas $e^{\tau / 2} W$ is in general unbounded for the three-parameter family of solutions for $\sqrt{3} / 2<\alpha<1$.

Next there are the solutions of Eqs (20), which turn out to be of interest only for $\beta>2$. As long as $r \partial V_{2} / \partial r<0$ there are two positive and two negative eigenvalues

$$
\gamma_{i}=-\frac{\kappa_{h}}{2} \pm \sqrt{\frac{\kappa_{h}^{2}}{4}+W_{h}^{2}+\frac{\beta^{2}}{2} r_{h}^{2} H_{h}^{2} \pm \sqrt{\left(W_{h}^{2}-\frac{\beta^{2}}{2} r_{h}^{2} H_{h}^{2}\right)^{2}+8 W_{h}^{2} r_{h}^{2} H_{h}^{2}}},
$$

i.e., the stable manifolds for $\tau \rightarrow \pm \infty$ are both three dimensional. For given $\alpha$ and $\beta$ and each set of values $\left(r_{h}, W_{h}, H_{h}\right)$ such that Eqs (20) are satisfied there exists a two-parameter family of local solutions with a degenerate horizon approaching the fixed point as $\tau \rightarrow-\infty$, and there exists a two parameter family of local solutions approaching the fixed point as $\tau \rightarrow+\infty$.

For $W=1$ and $H=0$ there is no fixed point since $r \partial V_{2} / \partial r>0$. Finally, for $W=H=0$ and as long as $r \partial V_{2} / \partial r<0$ all four eigenvalues $\gamma_{i}$ have a negative real part. There exists only one degenerate black hole solution, the extremal RN solution in a de Sitter background with $W \equiv H \equiv 0$, and $\kappa \rightarrow-\infty, N \rightarrow 0$ for some finite $\tau$.

The degenerate horizons for the theory with $\beta=\infty$ are similar to those for finite $\beta$. Discarding the two rows and columns corresponding to $H$ and $V$ and substituting $H=\alpha$ in the matrix $M$ (Eq. (27)) we obtain four eigenvalues $\kappa_{h},-2 \kappa_{h}$, and $\gamma_{1,2}$. There are, however, just two possible fixed points.

For the fixed point $r=\kappa=1, W=0$ the eigenvalues $\gamma_{1,2}$ are given by Eq. (28a) above. For $\alpha>1$ there exists a one-parameter family of local solutions with a degenerate horizon, for $\alpha<1$ there exists only the extremal RN solution with $W \equiv 0$. In addition for $\alpha>1$, resp. $\alpha<1$ there exists a one-, resp. two-parameter family of local solutions approaching the fixed point as $\tau \rightarrow+\infty$ and again $W$ oscillates with an exponentially decreasing amplitude for $\alpha<\sqrt{3} / 2$.

For the fixed point $r^{2}=1-W^{4}, \alpha^{2} r^{2}=\left(1-W^{2}\right), \kappa=\alpha^{2} r$ there is one positive and one negative eigenvalue

$$
\gamma_{1,2}=-\frac{\kappa_{h}}{2} \pm \sqrt{\frac{\kappa_{h}^{2}}{4}+2 W_{h}^{2}} .
$$

Therefore there exists a one-parameter family of local solutions with a degenerate horizon and a one-parameter family of local solutions approaching the fixed point as $\tau \rightarrow+\infty$.

The general results about the existence of a stable manifold require that none of the eigenvalues have a vanishing real part. This is no longer the case for a degenerate horizon with $\kappa_{h}=0$, e.g., at the point on the curve (21) where $\alpha$ has its minimal value. We are interested in particular in the case $\beta=\infty$ where this point $r_{h}=0, W_{h}=1, \alpha=1 / \sqrt{2}$ can also be seen as limiting case of the quasi regular solutions. Guided by a Taylor series expansion we introduce

$$
X_{ \pm}=\frac{W-1}{r^{2}} \pm \frac{U}{\sqrt{2} r}+\frac{1}{4} \mp \frac{r}{\sqrt{96}}, \quad \bar{N}=\frac{\sqrt{12} N}{r^{2}}-\frac{1}{r}, \quad \bar{\kappa}=\frac{\sqrt{3} \kappa}{r^{2}}-\frac{1}{r},
$$


and use the constraint (12) to express $\bar{\kappa}$ in terms of $X_{ \pm}, \bar{N}$ and $r$. The resulting differential eqs. are $\left(\right.$ with $\left.^{\prime} \equiv d / d r\right)$

$$
X_{ \pm}^{\prime}=\left(-\frac{2}{r} \pm \frac{\sqrt{24}}{r^{2}}\right) X_{ \pm}+f_{ \pm}, \quad \bar{N}^{\prime}=-\frac{4}{r} \bar{N}+f_{0} .
$$

The 'non-linear terms' $f_{ \pm, 0}$ as well as $\bar{\kappa} / r$ are bounded as long as $r$ is small and $X_{ \pm} / r$, $\bar{N} / r$ are bounded. In order to show that there exists a one-parameter family of solutions of the non-linear system (32) we closely follow the treatment for the standard case (25) in 13] p.330ff. The linear system obtained by substituting $f_{ \pm, 0}=0$ in Eqs. (32) has the solutions

$$
X_{+(0)}(r)=\left(\frac{r_{0}}{r}\right)^{2} e^{-\sqrt{24}\left(\frac{1}{r}-\frac{1}{r_{0}}\right)} X_{+}\left(r_{0}\right), \quad X_{-(0)}(r)=\bar{N}_{(0)}(r)=0
$$

with $X_{+}\left(r_{0}\right)$ as a free parameter. A solution of the non-linear system with the same boundary conditions $X_{+}\left(r_{0}\right)$ and $X_{-}(0)=\bar{N}(0)=0$ satisfies the integral equations

$$
\begin{aligned}
X_{+}(r) & =X_{+(0)}(r)-\int_{r}^{r_{0}}\left(\frac{t}{r}\right)^{2} e^{-\sqrt{24}\left(\frac{1}{r}-\frac{1}{t}\right)} f_{+}(t) d t \\
X_{-}(r) & =\int_{0}^{r}\left(\frac{t}{r}\right)^{2} e^{-\sqrt{24}\left(\frac{1}{t}-\frac{1}{r}\right)} f_{-}(t) d t \\
\bar{N}(r) & =\int_{0}^{r}\left(\frac{t}{r}\right)^{4} f_{0}(t) d t .
\end{aligned}
$$

Iterating these integral eqs. with the solution (33) of the linearized system as zeroth approximation one can easily estimate that the successive approximation converge to a solution of Eqs. (32) provided $r_{0}$ and $X_{+}\left(r_{0}\right)$ are sufficiently small. Apart from showing the existence of a one-parameter family of local solutions this method also allows to compute the solutions numerically by iterating a suitably discretized version of the integral Eqs. (34).

Let us finally consider the limit $r \rightarrow \infty$ with the two-parameter family (23,24a) of asymptotically flat solutions of Eq. (11). Here we introduce

$$
m(r)=\frac{r}{2}\left(1-N^{2}\right), \quad s(r)=r+m(r) \ln r, \quad Y_{ \pm}(r)=e^{\alpha s(r)}\left(W \mp \frac{U}{\alpha}\right)
$$

where $m$ is monotonously increasing and $M=m(\infty)$ is the total mass. Expressing $\kappa$ in terms of $m, Y_{ \pm}$, and $r$ we obtain the differential eqs. (again with ${ }^{\prime} \equiv d / d r$ )

$$
m^{\prime}=f_{0}, \quad Y_{+}^{\prime}=f_{+}, \quad Y_{-}^{\prime}=2 \alpha s^{\prime} Y_{-}+f_{-},
$$

with 'non-linear terms' $f_{0}$, resp. $f_{ \pm}$decreasing at least as $r^{-2}$, resp. $r^{-2} \ln r$ as long as $m$ and $Y_{ \pm}$are bounded. Together with the boundary conditions $m(\infty)=M, Y_{+}(\infty)=C, Y_{-}(\infty)=0$ we obtain the integral equations

$$
\begin{aligned}
m(r) & =M-\int_{r}^{\infty} f_{0}(t) d t \\
Y_{+}(r) & =C-\int_{r}^{\infty} f_{+}(t) d t \\
Y_{-}(r) & =-\int_{r}^{\infty} e^{-2 \alpha(s(t)-s(r))} f_{-}(t) d t .
\end{aligned}
$$

Iteration of these integral eqs. with $m_{(0)} \equiv M, Y_{+(0)} \equiv C$, and $Y_{-(0)} \equiv 0$ as zeroth approximation yields a convergent series for any given $M$ and $C$ and sufficiently large $r$. Note that in Eqs. (35, 36) we have used $s(r)=r+m(r) \ln r$ and not $\sigma(r)=r+M \ln r$ as in Eqs. (24). This allows to 
rewrite the integral eqs. in a form more suitable for our numerical procedure: Given $m\left(r_{0}\right)$ and $Y_{+}\left(r_{0}\right)$ for some sufficiently large value $r_{0}$, a slightly modified version of Eqs. (36) allows to compute $Y_{-}\left(r_{0}\right)$ as well as the asymptotic parameters $M$ and $C$.

The method described above can be easily adapted to the three-parameter family of asymptotically flat solutions with finite $\beta$ as long as $\beta<2$. The situation is, however, slightly different for $\beta \geq 2$, where the asymptotic behaviour of the Higgs field is dominated by non-linear terms. There still exists a three-parameter family of solutions but at least one of the parameters must be determined away from the singular point $r=\infty$ by an integral eq. of the type (34a).

\section{Global Existence for $\beta=\infty$}

In this section we shall discuss the global behaviour of solutions of the theory with $\beta=\infty$ (frozen Higgs field) whose field equations (11) were derived in Sect. 2. We restrict our discussion to solutions with either 'quasi regular' boundary conditions at $r=0$ (requiring $\alpha \leq 1 / \sqrt{2}$ ) or those for black holes. These solutions depend on one, resp. two parameters, determining their global behaviour.

Similarly to the case of the pure YM theory $(\alpha=0)$ discussed in [6] the global behaviour of the solutions can be characterized by the function $N(\tau)$. Since $N$ is positive close to the boundary point $\left(r=0\right.$ or $r=r_{h}$ ) it will either stay positive for all $\tau$ or have a zero for some finite $\tau_{0}$. In the latter case, which turns out to be the generic one, $N$ has to change sign (a zero of even order leads to $N \equiv 0$ ). From Eq. (11) a) it follows that $r$ has a maximum at $\tau_{0}$. Since $N$ stays negative for $\tau>\tau_{0}$ the function $r$ runs back to $r=0$, where the solution develops a curvature singularity. The corresponding spaces $t=$ const. are compact singular 3-manifolds.

In the case when $N$ stays positive two possibilities arise. Either $r$ grows without bound or it tends to some finite limit. The former case yields the globally (quasi) regular, resp. black hole solutions. In the latter case the solution runs into the fixed point $N=0, r=1, W=0$. The $t=$ const. hypersurfaces are non-compact developing a 'cylindrical throat' for $\tau \rightarrow \infty$ similar to the extremal RN solution for $\tau \rightarrow-\infty$. However in contrast to the latter the metrical function $A$ diverges for $\tau \rightarrow \infty$. Thus this solution does not represent the interior of an extremal black hole.

The precise formulation of the described results is the content of Theorem 1. Its proof, which we have deferred to the Appendix, follows closely the one for the analogous Theorem 16 of [6], but requires some changes in technical details.

Theorem 1: For both types of boundary conditions at $r=0$, resp. $r=r_{h}$ the global behaviour of solutions of Eqs. (11) is completely classified by the following three cases:

i) $N(\tau)$ changes sign for some finite value of $\tau$, i.e., $r(\tau)$ attains a maximum. Then $N$ stays negative and $r$ turns back to 0 . This leads to a compact singular 3-space ('bag of gold').

ii) $N$ stays positive and tends to 1 for $\tau \rightarrow \infty$ and hence $r \rightarrow \infty$. In this limit $W \rightarrow 0, U \rightarrow 0$ and $\kappa \rightarrow 1$. These are the quasi regular, resp. black hole solutions.

iii) $N$ stays positive and tends to 0 for $\tau \rightarrow \infty$. The solution approaches the fixed point $N=U=W=0, r=\kappa=1$ discussed in the preceding section. There it was shown, that for $0 \leq \alpha<\sqrt{3} / 2$ the function $W(\tau)$ oscillates, whereas for larger values of $\alpha$ the behaviour is exponential for $\tau \rightarrow \infty$.

The main result based on the above classification is the existence of quasi regular, resp. black hole solutions of Eqs. (11). Already from the initial conditions at $r=0$ it follows that quasi regular solutions can exist only for $0 \leq \alpha \leq 1 / \sqrt{2}$. Similarly one obtains a certain bounded domain in the $\left(\alpha, r_{h}\right)$ plane, where black hole solutions exist. Although parts of the boundary 
of this domain are known only through numerical calculations, we are able to establish its qualitative features with analytical methods. Similarly to the case $\alpha=0$ one obtains discrete families of solutions, whose members are distinguished by the number of zeros of the function $W$.

The precise formulation of our 'Existence Theorem' is

\section{Theorem 2:}

1. For any $0 \leq \alpha \leq 1 / \sqrt{2}$ and any integer $n \geq 0$, there exists at least one global quasi regular solution with $n$ zeros of $W$. For the same values of $\alpha$ there also exists at least one 'oscillating solution' with infinitely many zeros of $W$ and $0 \leq r<1$ whose asymptotic behaviour is given in Eqs. (29).

2.a For any $0<r_{h}<1, \alpha<\min \left(\frac{\sqrt{3}}{2}, \alpha_{\mathrm{e}}\left(r_{h}\right)\right)$, and $n \geq 0$, there exists at least one global black hole solution with $n$ zeros of $W$. For $\alpha=\alpha_{\mathrm{e}}\left(r_{h}\right), 0<r_{h}<2 \sqrt{2} / 3$ corresponding black holes with a degenerate horizon exist. Under the same assumptions for $r_{h}$ and $\alpha$ there exists at least one 'oscillating solution' with infinitely many zeros of $W$ and $r_{h} \leq r<1$ ).

$2 . b r_{h} \geq 1$ : For $0 \leq \alpha<\sqrt{3} / 2$ and for any $n \geq 0$ there is some $r_{h, \max }(\alpha, n)>1$ such that black hole solutions with $n$ zeros of $W$ exist for $1 \leq r_{h}<r_{h, \max }(\alpha, n)$. Furthermore $r_{h, \max } \rightarrow \infty$ for $\alpha \rightarrow 0$, i.e., there exist black holes for arbitrarily large $r_{h}$ if $\alpha$ is taken small enough.

Remark: For $\alpha<\sqrt{3} / 2$ one may consider the limit of asymptotically flat (quasi regular or black hole) solutions when $n$ tends to $\infty$. Using the arguments of [6, 15] one can show that (a suitable subsequence of) these solutions converge pointwise to an oscillating solution for $r<1$ and to the extremal RN black hole for $r>1$.

Remark: There is convincing numerical evidence that for $\alpha \geq \sqrt{3} / 2$ no black hole solutions exist. (Compare Fig. 6).

The proof of Theorem 2 consists of two main parts. First we demonstrate the existence of solutions with a regular horizon, following closely the analogous discussion in [6]. We then show that the quasi regular solutions as well as the extremal black holes are obtained as limits of regular black holes. This approach is necessary since the arguments of [6] cannot be applied directly to these cases. It also shows how the regular black hole solution tend to, e.g., quasi regular ones as $r_{h} \rightarrow 0$.

The existence proof for regular black holes can be shortly summarized as follows: First we partition the initial data into sets corresponding to the three cases introduced in Theorem 1 and the number of zeros of $W$. Using the smooth dependence on initial data we establish that the sets $\mathbf{R e g}_{n}$ corresponding to asymptotically flat solutions with $n$ zeros (Case ii) must lie between open sets Sing $_{n}$ or Sing Sit1 $_{n}$ corresponding to 'singular' solutions (Case i). Analogous results hold for solutions corresponding to Case iii. Knowing that the open sets $\mathbf{S i n g}_{n}$ or $\mathbf{S i n g}_{n+1}$ are not empty we conclude that there exist initial data in $\mathbf{R e g}_{n}$.

We have seen in Sects. 3 and 1 that local solutions with a regular horizon exist as long as the condition $N_{1}>0$ is satisfied (compare Eq. (19)). For any $\alpha$ these initial data form a subset of the $\left(r_{h}, W_{h}\right)$-plane shown as region $\mathrm{C}$ in Fig. 2; for given $\alpha$ and $r_{h}$ there are at most two $W_{h}$-intervals.

Excluding the RN black holes (14) with $W \equiv 0$ and using the invariance of Eqs. (11) under the 'reflection' $(W, U) \rightarrow(-W,-U)$ we need only consider initial data with $W_{h}>0$. Based on the classification in Theorem 1 and using some results from its proof we can partition the set of initial data with $N_{1}>0$ and $W_{h}>0$ into various subsets as follows.

For Case $\mathrm{i}$ there is some $\tau_{0}$ such that $N\left(\tau_{0}\right)=0$ and a corresponding point $\left(r_{0}, W_{0}\right)=$ $\left(r\left(\tau_{0}\right), W\left(\tau_{0}\right)\right)$ in the $(r, W)$-plane. We introduce $\mathbf{S i n g}_{n}, n=0,1, \ldots$ as the set of initial data 
such the zero of $N$ is in region B of Fig. 2 where $W_{0}^{2}+\alpha^{2} r_{0}^{2}>1$ and $W$ has $n$ zeros. Sing is $_{\infty}$ the set of initial data such that the zero of $N$ is in region A where $W_{0}^{2}+\alpha^{2} r_{h}^{2}<1$.

For Case ii we let $\operatorname{Reg}_{n}, n=0,1, \ldots$ be the set of initial data for regular, asymptotically flat black holes with $n$ zeros of $W$.

Finally we denote the set of initial data corresponding to Case iii by Osc for $\alpha<\sqrt{3} / 2$ and $\operatorname{Exp}$ for $\alpha \geq \sqrt{3} / 2$. Solutions with initial data in Osc have infinitely many zeros of $W$, i.e., oscillate. Solutions with initial data in Exp have finitely many zeros and we can further partition the set $\operatorname{Exp}$ into sets $\mathbf{E x p}_{n}$ according to this number of zeros. As discussed in Sect. 1 , $e^{\tau / 2} W$ is unbounded for generic initial data in $\operatorname{Exp}$ when $\alpha<1$. There is, however, a subset in Exp, corresponding to a lower dimensional stable manifold such that $e^{\tau / 2} W$ remains bounded.

Let us next study the change in the behaviour of solutions induced by a small change in the initial data, exploiting the continuous dependence of the solutions on their initial data.

Case $\mathrm{i}$ is generic in the sense that it is stable under sufficiently small changes in the initial data. Moreover, for a zero of $N$ in region B the number of zeros of $W$ does not change as long as $W_{h}>0$. Therefore Sing $_{n}$ and Sing $_{\infty}$ are open sets.

For solutions with initial data in $\boldsymbol{R e g}_{n}$ the $n$ zeros of $W$ occur for $r<1 / \alpha$ and $|W|$ monotonously decreases when $r>1 / \alpha$. Given any $r_{1} \gg 1 / \alpha$, the properties of the solution are unchanged for $r \leq r_{1}$ under a sufficiently small change of the initial data. The function $|W|$ may, however, start to increase for $r>r_{1}$ with or without an additional zero of $W$. Therefore a sufficiently small neighbourhood of a point in $\mathbf{R e g}_{n}$ consists of points in $\mathbf{R e g}_{n}$, Sing ${ }_{n}$, or $\operatorname{Sing}_{n+1}$.

Solutions with initial data in Osc or Exp satisfy $N>0$ and $r<1$ for all $\tau$ and furthermore $W \rightarrow 0$ as $\tau \rightarrow \infty$. Given any $\tau_{1} \gg 1$, these inequalities remain valid for $\tau \leq \tau_{1}$ for a sufficiently small change of the initial data, They may, however, be violated for some $\tau>\tau_{1}$ with either $N=0$ and $r<1$ or $N>0$ and $r=1$ while $|W| \ll 1$. Given initial data in Osc and any positive integer $n$, a sufficiently small change of the initial data yields a solution with at least $n$ zeros of $W$. Therefore a sufficiently small neighbourhood of a point in Osc consists of points in Osc, Sing $_{\infty}$, or $\bigcup_{m \geq n}\left(\boldsymbol{R e g}_{m} \cup\right.$ Sing $\left._{m}\right)$.

The analysis for Exp is slightly more complicated. A sufficiently small neighbourhood of a generic point in $\mathbf{E x p}_{n}$ consists of points in $\mathbf{E x p}_{n}, \mathbf{S i n g}{ }_{\infty}$, or $\mathbf{S i n g}_{n}$. This result is partly based on the observation that solutions missing the fixed point have no zeros of $W$ for $r>1$, a fact directly related to the stability of the RN solution when $\alpha>\sqrt{3} / 2$ discussed in Sect. 77. The neighbourhood of a point in $\mathbf{E x p}_{n}$ for which $e^{\tau / 2} W$ is bounded can in addition contain points in $\boldsymbol{R e g}_{n}, \mathbf{E x p}_{n+1}$, or $\mathbf{S i n g}_{n+1}$.

In analogy to Lemma 21 and 24 of [6] one can show that for given $\alpha$ and $r_{h}$ each of the sets $\boldsymbol{R e g}_{n}$ or Osc, resp. Exp consists at most of isolated points.

Consider a continuous one-parameter family of initial data for some $\alpha<1$, e.g., a $W_{h}$-interval with $r_{h}$ fixed. From the above results we can immediately draw the following conclusions:

1. A family interpolating between Sing $_{\infty}$ and Sing $_{0}$ contains at least one point in Osc, resp. $\operatorname{Exp}$ for $\alpha<\sqrt{3} / 2$, resp. $\alpha \geq \sqrt{3} / 2$.

2. A family interpolating between Osc and $\mathbf{S i n g}_{0}$ contains at least one point in each $\mathbf{R e g}_{n}$.

3. A family interpolating between $\mathbf{E x p}_{n}$ or $\mathbf{S i n g}_{n}$ and $\mathbf{S i n g}_{0}$ contains at least one point in each $\boldsymbol{R e g}_{m}$ for $0 \leq m<n$.

We now use the above results for families connecting points near the boundary of the domain of initial data with $N_{1}>0$ and $W_{h}>0$. For $\alpha<1$ this boundary consists of three pieces:

a) The part of the quartic curve $N_{1}=0$ inside the ellipse $W_{h}^{2}+\alpha^{2} r_{h}^{2}=1$, i.e., the boundary between regions $\mathrm{C}$ and $\mathrm{A}$ of Fig. 2 . 
b) The part of the quartic curve outside the ellipse, i.e., the boundary between regions $\mathrm{C}$ and B.

c) The line $W_{h}=0, r_{h} \geq 1$.

For initial data sufficiently close to the curve $N_{1}=0$ we can use the truncated version of Eqs. (18) corresponding to $\beta=\infty$ and deduce that there must be a zero of $N$ for some $\tau \ll 1$ with $r \approx r_{h}$ and $W \approx W_{h}$. Therefore initial data close to the boundary between regions $\mathrm{C}$ and $\mathrm{A}$ are in $\mathbf{S i n g}_{\infty}$ and those close to the boundary between regions $\mathrm{C}$ and B are in $\mathbf{S i n g}_{0}$. Actually all boundary conditions outside the ellipse, i.e., in region $\mathrm{C}_{-}$, are in $\mathbf{S i n g}_{0}$ because $W_{h} \neq 0$ implies $U W>0$ for all $\tau>0$. This is true in particular for all boundary conditions for $\alpha \geq 1$ except those for the RN solution with $W \equiv 0$. This concludes the existence proof for regular black holes with $r_{h}<1$.

In order to prove the existence of regular black holes with $n$ zeros of $W$, i.e., that $\mathbf{R e g}_{n}$ is not empty for given $\alpha$ and $r_{h} \geq 1$, it is sufficient to show that solutions with $W_{h} \approx 0$ have at least $n+1$ zeros, i.e., have initial data in $\bigcup_{m>n}\left(\mathbf{R e g}_{m} \cup \mathbf{S i n g}_{m}\right)$. Solutions with $r_{h}>1$ remain close to the RN black hole (14) on any finite $r$-interval if $W_{h}$ is chosen small enough.

We first want to prove the existence of regular black holes for any $r_{h}>1$ and $n$. Given $r_{h}$ and $n$ we choose $\alpha \ll 1 / r_{h}$. For $r_{h} \ll r \ll 1 / \alpha$ we may use the approximate form $W \approx$ $C \sqrt{r} \sin \left(\frac{\sqrt{3}}{2} \ln r+\theta\right)$ valid for $N \approx 1 \approx \kappa$ and $W \approx 0$ to argue that the solution has at least $n+1$ zeros.

Finally we consider regular black holes for given $\alpha<\sqrt{3} / 2$ and $n$. Choosing initial data near the curve $N_{1}=0$ (i.e., $\max \left(r_{h}-1, W_{h}^{2}\right) \ll 1$ ) the solution comes close to the fixed point $r=\kappa=1, N=W=U=0$ and stays there long enough to have at least $n+1$ zeros. This can be deduced from the approximate form of $W$ given in Eq. (29). This concludes the existence proof for regular black holes with $r_{h} \geq 1$.

In the proof of existence for regular black hole solutions we have followed the same strategy as in [6] which indeed went through with some minor changes in the arguments. Trying to extend this stragegy to the quasi regular solutions and extremal black holes we encounter difficulties. An essential part of this strategy has been to determine the behaviour of solutions with initial data close to the boundary of their allowed region. Whereas for $\alpha=0$ the regular solutions behave as $W \approx 1-b r^{2}$ near $r=0$, this changes to $W \approx 1-r^{2} / 4+\ldots+b r^{\gamma}$ with $\gamma>2$ for the quasi regular solutions with $0<\alpha<1 / \sqrt{2}$ (compare Eq. (17)). The method used in [6] to analyze the behaviour of solutions with large $b$ is not directly applicable for $\alpha>0$. This is particularly evident for $\alpha \rightarrow 1 / \sqrt{2}$ where $\gamma \rightarrow \infty$. A similar situation is encountered for black holes with a degenerate horizon.

On the other hand it turns out to be possible to obtain the quasi regular solutions and extremal black holes as limits of regular black holes. Having established the global existence of (asymptotically flat and oscillating) regular black holes, we shall now consider the limiting cases corresponding to points on the boundary of the allowed domain in the $\left(\alpha, r_{h}\right)$ plane.

Guided by the numerical results we shall prove that regular black hole solutions with $r_{h} \ll 1$ consist of two pieces separated by a large $\tau$-interval: Starting at the horizon with $N=0$ the solution first approaches the fixed point $N=\kappa=\sqrt{1-2 \alpha^{2}}, W=1, U=r=0$. Since, however, $r>0$ the solution necessarily misses this fixed point and eventually runs away along the stable manifold for $\tau \rightarrow-\infty$ corresponding to the quasi regular solutions. In the limit $r_{h} \rightarrow 0$ the two pieces get separated by an infinite $\tau$-interval and the outer part lies on the stable manifold. The same type of argument was used in [15] to show that the Bartnik-McKinnon solutions converge to the extremal RN solution for $r>1$ as the number of zeros of $W$ tends to $\infty$.

In order to construct a quasi regular monopole with $n$ zeros of $W$ for some $\alpha<1 / \sqrt{2}$ we take a sequence of regular black holes with $n$ zeros and $r_{h} \rightarrow 0$. Since the values $\left(W_{0}, U_{0}, N_{0}, \kappa_{0}\right)$ at some regular point $r=r_{0}$ are uniformly bounded we can select a convergent subsequence. In 
order to obtain a convergent sequence of solutions of the autonomous system (11) we have to adjust the variable $\tau$. For that purpose we introduce a shifted variable $\bar{\tau}=\tau-\tau_{0}\left(r_{h}\right)$ where $\tau_{0}$ is determined by $r\left(\tau_{0}\right)=r_{0}$. In view of the uniqueness theorem for the initial value problem at a regular point the sequence of solutions converges for fixed $\bar{\tau}$ to a limiting solution defined for all $r>0$, representing the outer part mentioned above.

In order to study the inner part we consider the solutions for fixed $\tau$. Introducing $\rho=r / r_{h}$ and $T=(W-1) / r$ we obtain from Eqs. (11, 12):

$$
\begin{aligned}
\dot{\rho} & =\rho N, \\
\dot{N} & =\frac{1}{2}\left(1-2 \alpha^{2}-N^{2}-2 U^{2}-4 T^{2}\right)+O\left(r_{h}\right), \\
\dot{\kappa} & =1-2 \alpha^{2}-\kappa^{2}+2 U^{2}+O\left(r_{h}\right), \\
\dot{T} & =U-N T, \\
\dot{U} & =2 T-(\kappa-N) U+O\left(r_{h}\right) .
\end{aligned}
$$

The geometrical structure of the allowed domain of black hole boundary conditions for $r_{h}$ close to zero (see Fig. 1) implies the existence of a uniform bound for the the initial values $T_{h}=T(0)$ at the horizon. Hence way may select a further subsequence for which the values $T_{h}$ converge to some $\bar{T}$, the initial value of the limiting solution. We will show that $\bar{T}$ must be zero because otherwise $N$ would vanish for some finite $\tau$. In the limit $r_{h} \rightarrow 0$ Eqs. (37) imply

$$
(T U)^{\circ}=2 T^{2}+U^{2}-\kappa T U \geq(\sqrt{8}-\kappa)|T U| .
$$

Assuming $\bar{T} \neq 0$ we conclude that $T U$ grows exponentially as soon as $\kappa<\sqrt{8}$, because $T U>0$ for $\tau>0$. Using the Schwarz inequality it follows from Eq. (37 b) that $N$ must vanish for some large enough $\tau$, contradicting the properties of the limiting solution. Therefore $\bar{T}=0$ and hence $T \equiv U \equiv 0$. Solving the remaining equations we conclude that the limiting solution tends to the fixed point $N=\kappa=\sqrt{1-2 \alpha^{2}}, W=1, U=r=0$ for $\tau \rightarrow \infty$.

The remaining argument closely follows the one used in 15. The solutions for sufficiently small $r_{h}$ come arbitrarily close to the fixed point, miss it, and run away along the stable (for $\tau \rightarrow-\infty)$ manifold. In a small but fixed neighbourhood of the fixed point the distance from the stable manifold decreases exponentially with increasing $\tau$. This implies that in this small neighbourhood the outer part of the limiting solution lies on the stable manifold, i.e., is a quasi regular solution.

The arguments for the existence of extremal black holes are fairly similar to those for quasi regular solutions described above. The values of $r, W$, and $\kappa$ at a degenerate horizon for $1 / \sqrt{2}<\alpha<1$ are

$$
r_{e}=\frac{\sqrt{2 \alpha^{2}-1}}{\alpha^{2}}, \quad W_{e}=\frac{\sqrt{1-\alpha^{2}}}{\alpha}, \quad \kappa_{e}=\sqrt{2 \alpha^{2}-1},
$$

and $\left(W_{h}-W_{e}\right) / \sqrt{r_{h}-r_{e}}$ is uniformly bounded. This suggests to introduce new variables $\rho=$ $\left(r-r_{e}\right) /\left(r_{h}-r_{e}\right), n=N /\left(r-r_{e}\right), w=\left(W-W_{e}\right) / \sqrt{r_{e}\left(r-r_{e}\right)}$, and $u=r U / \sqrt{r_{e}\left(r-r_{e}\right)}$. Given a sequence of (asymptotically flat or oscillating) non-degenerate black holes with $r_{h} \rightarrow r_{e}$ we can select a subsequence such that $\left(W_{0}, U_{0}, N_{0}, \kappa_{0}\right)$ at some regular point $r_{0}>r_{e}$ as well as $w_{h}$ converge. Rewriting Eqs. (11) in terms of the rescaled variables $\rho, n, w$, and $u$ and neglecting contributions that vanish as $r_{h} \rightarrow r_{e}$ we obtain

$$
\begin{aligned}
& \dot{\rho}=\rho n, \\
& \dot{n}=(\kappa-n) n-2 u^{2}, \\
& \dot{\kappa}=\kappa_{e}^{2}-\kappa^{2},
\end{aligned}
$$




$$
\begin{aligned}
& \dot{w}=-\frac{1}{2} n w+u, \\
& \dot{u}=2 W_{e}^{2} w-\left(\kappa+\frac{1}{2} n\right) u,
\end{aligned}
$$

with the constraint

$$
\kappa n=\kappa_{e}^{2}+u^{2}-2 W_{e}^{2} w^{2} .
$$

Solving Eqs. (39) with initial data $\rho(0)=1$ and $w(0)=w_{h}$, we first observe that $\kappa \rightarrow \kappa_{e}$ and $n$ remains bounded as $\tau \rightarrow \infty$. Next we want to show that $w$ and $u$ remain bounded. Note that $w u>0$ for $\tau>0$ except when $w_{h}=0$ and hence $w \equiv 0 \equiv u$. For large $\tau$ when $\kappa \approx \kappa_{e}$ the linear system for $\sqrt{\rho} w$ and $\sqrt{\rho} u$ has the two eigenvalues

$$
\gamma_{1,2} \approx \frac{1}{2}\left(-\kappa_{e} \pm \sqrt{\kappa_{e}^{2}+8 W_{e}^{2}}\right)
$$

and therefore $\sqrt{\rho} w$ and $\sqrt{\rho} u$ both grow as $e^{\gamma_{1} \tau}$. This is, however, not possible if $2 \gamma_{1}>\kappa_{e}$ since $(\rho n)^{\circ}=\kappa \rho n-2 \rho u^{2}$ would then imply that $n$ vanishes for some finite $\tau$. Therefore $w$ and $u$ are either bounded (for $2 \gamma_{1} \leq \kappa_{e}$ ) or are identically zero (for $2 \gamma_{1}>\kappa_{e}$ ). We conclude that the inner part of the solution for $r_{h} \rightarrow r_{e}$ approaches the fixed point $r=r_{e}, W=W_{e}, \kappa=\kappa_{e}$, and $N=U=0$ as $\tau \rightarrow \infty$. The remaining step of the argument proceeds as above for the quasi regular solutions.

\section{Numerical Results}

The problem to find global solutions of Eqs. (9) by numerical integration is complicated by the singular nature of the boundary points. As was discussed in Section the regular solutions lie on submanifolds of the phase space, the 'stable manifolds' of these singular points. Hence the construction of global solutions may be reduced to finding intersections of the stable manifolds emanating from the two boundary points. This, however, requires to extend the stable manifolds from the neighbourhood of the boundary points to some common value of $r$. A general strategy to achieve this goal is the following. Close to the singular points one replaces the differential equations by a system of integral equations implementing the correct boundary conditions at the singular point [13]. The stable manifold is then parametrized by a subset of the boundary values at the other endpoint of integration. The integral equations, resp. suitable discretizations, may be solved by iteration (a procedure guaranteed to converge close enough to the singular points). The output of this first step is a set of initial data for the differential equations at regular points, which may then be integrated using, e.g., the Runge-Kutta method to some common value of $r$. Varying the free boundary values of the integral equations at either end one may try to intersect the two stable manifolds at this common value of $r$.

The procedure just described is admittedly rather complicated, yet certain simplifications are possible in most cases. Since the numerical integration procedures come with certain numerical errors anyway, one may as well approximate the stable manifolds close to the boundary points by their linearizations, provided this does not introduce unduely big errors. This allows to circumvent the use of the integral equation. Another case in which the integral equation can be avoided is, that the stable manifold can be directly parametrized at the singular point. In this case one may use the Runge-Kutta method immediately from the boundary point. Thus in the simplest case one starts, e.g., directly at $r=0$, resp. $r=r_{h}$ integrating the Eqs. (9) with the help of the Runge-Kutta method as close as possible to the other boundary point, e.g., $r=\infty$. Linearization close to this singular point results in unstable modes depending on the choice of the free boundary values at the starting point. Studying the effect of finite or infinitesimal variations of these values one can try to suppress iteratively the unwanted unstable modes, thus improving the solution. Cases where this simplified approach fails are extremal black holes, the 

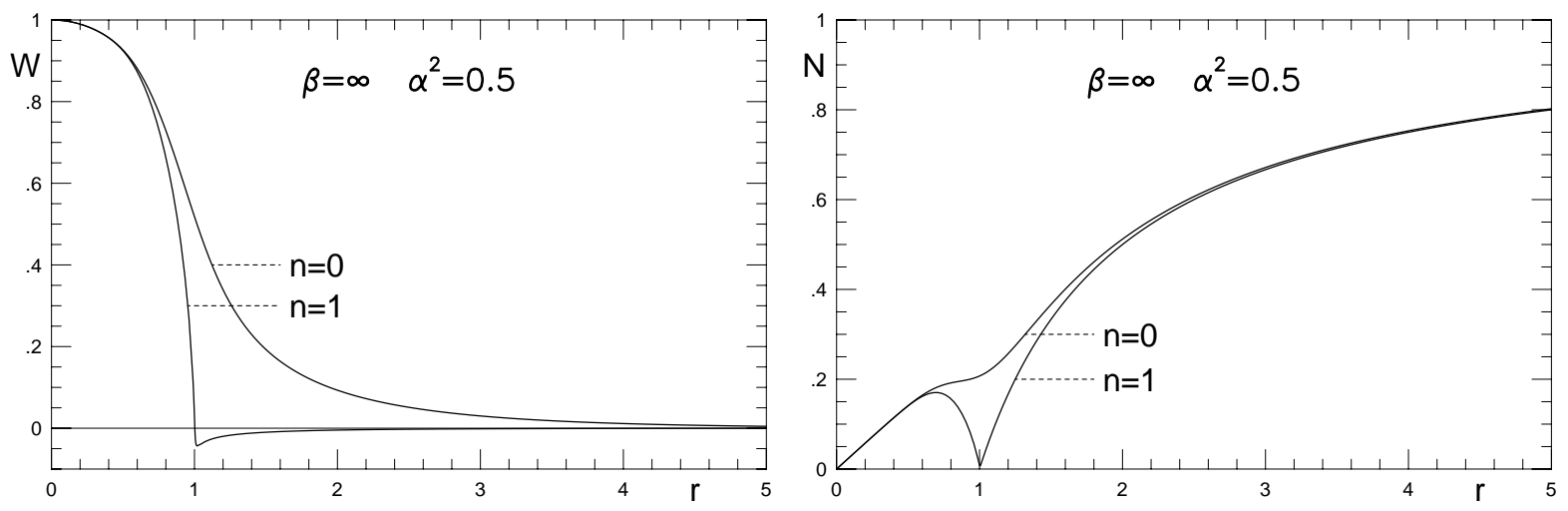

Figure 4: The fundamental and first excited monopole solution for $\beta=\infty$ and $\alpha=1 / \sqrt{2}$
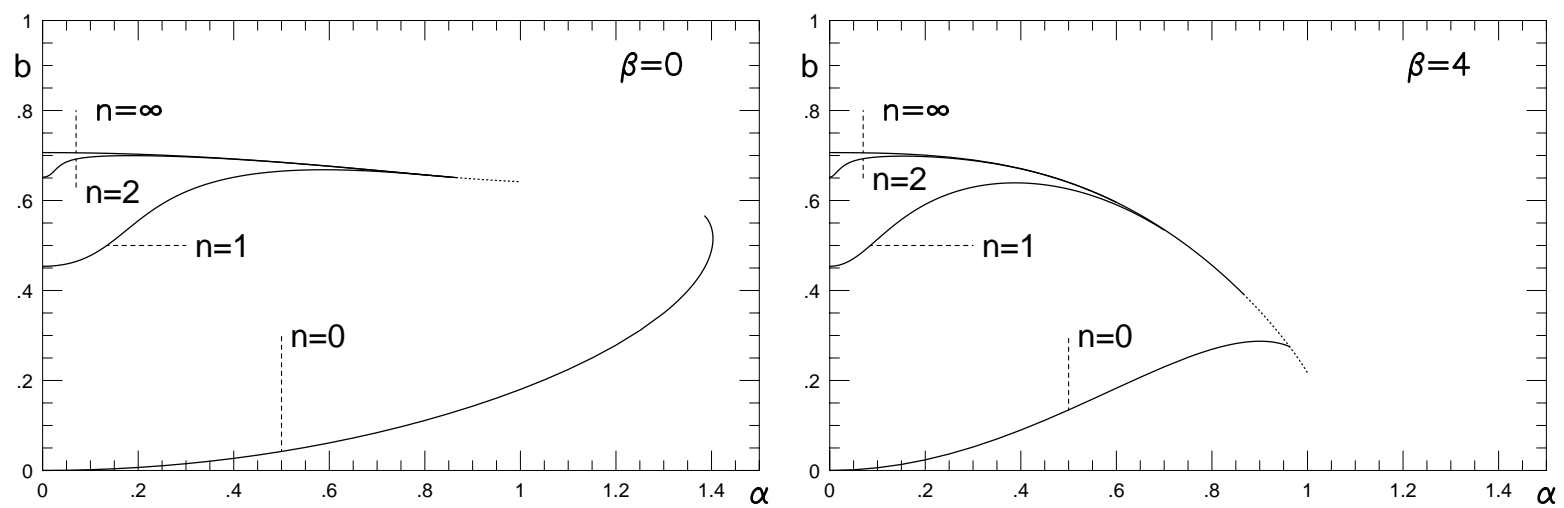

Figure 5: The parameter $b$ vs. $\alpha$ of the fundamental monopole, the first two excited ones, and the oscillating, resp. exponential solution for $\beta=0$ and $\beta=4$

'quasi regular' solution for $\alpha$ close to $1 / \sqrt{2}$ and solutions for large but finite values of $\beta$. The reason for this failure in the last case is the rapid growth of the unstable Higgs-mode $\left(\approx e^{\beta r}\right)$ before one reaches the asymptotic region for $W$.

Fig. 1 shows the fundamental and first excited quasi regular solution $(\beta=\infty)$ for the extreme case $\alpha=1 / \sqrt{2}$. These solutions could not be obtained without using integral equations to determine the stable manifold at the origin.

Let us now proceed to a qualitative description of the domain in the space of the parameters $\alpha, \beta, r_{h}$ and $W_{h}$ for which global black hole solutions exist. Starting from one particular solution one may vary these parameters and try to find a whole family of such solutions in a connected part of the parameter space. In view of the smooth dependence of the solutions on the parameters the boundary of the corresponding region in parameter space must be determined by some kind of singular behaviour. A typical phenomenon of this type is that the solutions on their way from $r=r_{h}$ to $r=\infty$ get caught by some fixed point different from $r=\infty$. This is indicated by the fact that the solutions spend more and more 'time' (in $\tau$ ) in the vicinity of this wrong fixed point. A second phenomenon is a change in the nature of the boundary condition at $r_{h}$. A trivial boundary of this type is given by the condition $r_{h} \geq 0$. Another case is the degeneracy of the horizon.

Beginning with the case $\beta=0$ already described in some detail in I, it is instructive to observe what happens with the fundamental monopole, if one starts from the flat space PrasadSommerfield solution and increases $\alpha$ (in order to obtain the flat limit a rescaling $r \rightarrow r / \alpha$ is required and hence $b(\alpha) \approx \alpha^{2} / 6$ for small $\alpha$ ). As seen from Fig. 5 the parameter $b$ increases 

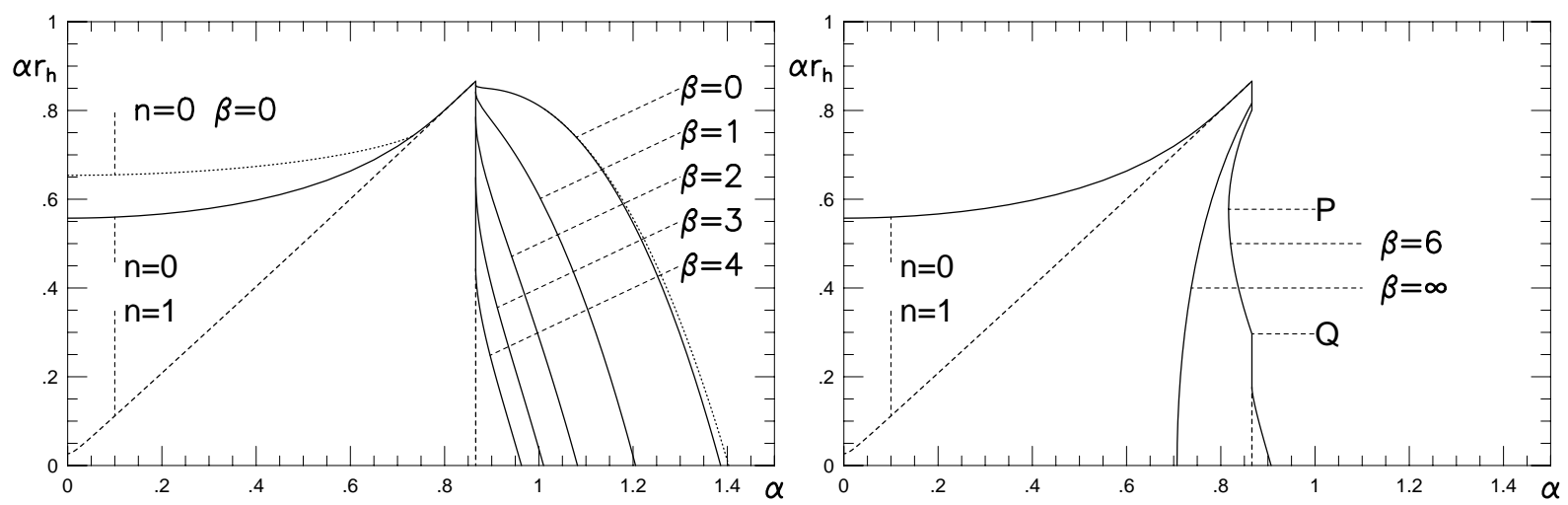

Figure 6: Domains of existence $\mathcal{E}$ for non-abelian black holes: a) for $\beta=0,1,2,3$, and 4 ; b) for $\beta=6$ and $\infty$

monotonously while $\alpha$ runs through a maximal value $\alpha_{\max }$. Finally the regular monopole ceases to exist for some critical value of $\alpha=\alpha_{c}$. At this point the solution runs into the 'wrong' fixed point with $N=0$ at $r=1$, i.e., bifurcates with a solution belonging to $\operatorname{Exp}$. For $\alpha$ between $\alpha_{c}$ and $\alpha_{\max }$ we have two different monopole solutions with a bifurcation point at $\alpha_{\max }$. As usual this bifurcation is accompanied by a change in stability. Whereas the solutions on the lower branch of the curve $b(\alpha)$ are stable against linear perturbations the ones on the upper one have exactly one unstable mode [16]. It is suggestive to relate this unstable mode to the gravitational instability observed for the Bartnik-McKinnon (BM) solution [17]. In fact, as seen from Fig. 5 there is a second branch of the $b(\alpha)$ curve for the first excited solution starting with the BM value $b \approx 0.4537$ for $\alpha=0$ and ending at the critical value $\alpha_{c}=\sqrt{3} / 2$. All the solutions on this branch have also one unstable mode. Similar curves exist for the higher excited solutions, all of them ending at $\alpha_{c}=\sqrt{3} / 2$ with the same value of $b\left(\alpha_{c}\right)$. At this common limiting point all the excited monopole solutions bifurcate with the solution in Osc, i.e., run into the fixed point with $N=0$ at $r=1$.

The same type of phenomena persists for black holes of small radius $r_{h}$. For given $r_{h}$ nonabelian black holes exist only for a finite $\alpha$-interval. The domain of existence $\mathcal{E}$ in the $\left(\alpha, r_{h}\right)$ plane is shown in Fig. 6 (where $\alpha r_{h}$ is plotted versus $\alpha$ ). The difference between $\alpha_{\max }$ and $\alpha_{c}$ for the fundamental solution decreases with increasing $r_{h}$ and disappears for some limiting value $r_{h} \approx 0.793$. Also the value of $\alpha_{c}$ decreases with increasing $r_{h}$ tending to $\alpha_{c}=\sqrt{3} / 2$ for $r_{h} \approx 0.991$ still below the maximal value of $r_{h}=1$. This means that the curve delimiting the domain $\mathcal{E}$ has a short straight section between the points $(\sqrt{3} / 2,0.991)$ and $(\sqrt{3} / 2,1)$. For $\sqrt{3} / 2<\alpha<1$ the points on the curve $\left(\alpha_{c}\left(r_{h}\right), r_{h}\right)$ are determined by the condition that the solution in Exp ceases to have a zero as $r_{h}$ is increased from zero. The reason is that at the critical value of $\alpha$ the monopole solution bifurcates with the solution in $\operatorname{Exp}$ with $e^{\tau / 2} W$ bounded (compare the discussion in the preceding section). For $\alpha=\sqrt{3} / 2$ this just happens at $r_{h} \approx 0.991$.

As was already explained in I the curve delimiting the existence domain $\mathcal{E}$ in the $r_{h}$ direction for $\alpha<\sqrt{3} / 2$ is determined by a different mechanism. As $r_{h}$ is varied (for some fixed value of $\alpha)$ the value for $W_{h}$ decreases from 1 to 0 . It turns out that the curve $\left(r_{h}, W_{h}\right)$ can be continued smoothly to negative values by the reflection $W_{h} \rightarrow-W_{h}$, i.e., $r_{h}$ runs through an extremum as $W_{h}$ is varied from positive to negative values. For small values of $\alpha$ the extremum of $r_{h}$ at $W_{h}=0$ turns out to be a minimum, i.e., the maximal value of $r_{h}$ occurs for some $\left|W_{h}\right|>0$ (compare Fig. 7). With increasing $\alpha$ the extremal points of $r_{h}$ approach each other and merge for some particular value of $\alpha \approx 0.732$. Qualitatively the same phenomenon repeats itself for all the excited solutions, the extremal point of $r_{h}$ with $W_{h}=0$ moving inwards towards $r_{h}=1$ with increasing order of the excitation. 

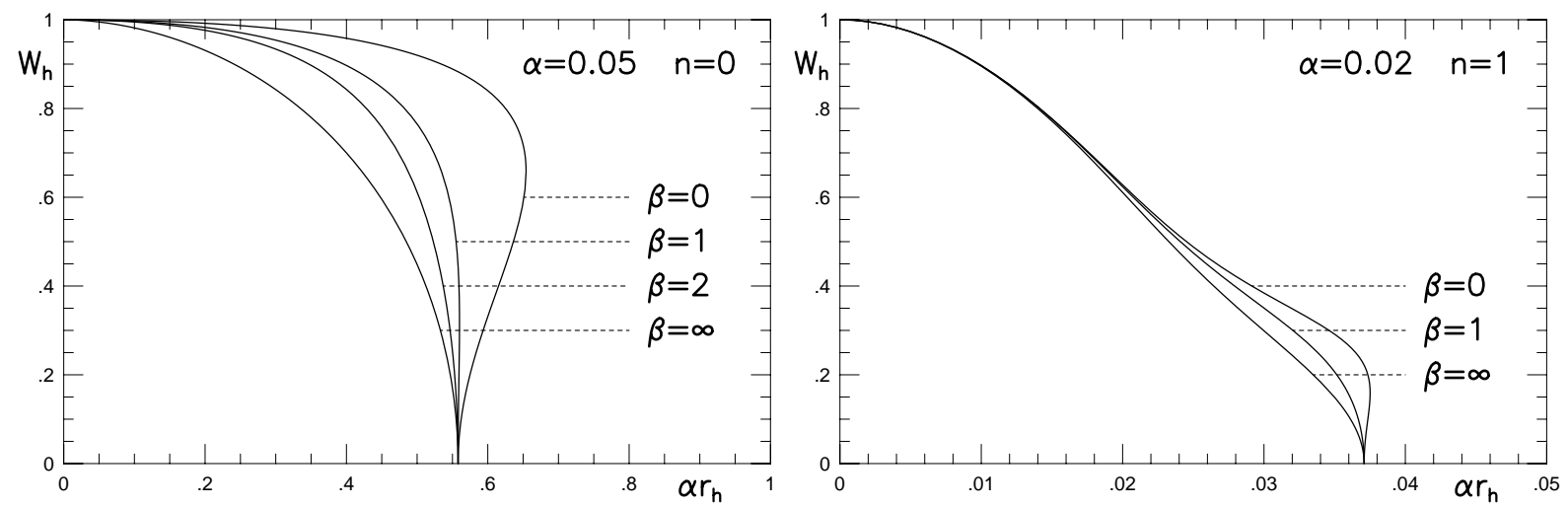

Figure 7: Initial data $r_{h}$ and $W_{h}$ for the fundamental and first excited black hole solution for $\alpha=0.05$, resp. $\alpha=0.02$ and various values of $\beta$

For $W_{h}=0$ the non-abelian black holes bifurcate with the (non-extremal) abelian RN black hole. As $\alpha$ tends to $\sqrt{3} / 2$ the corresponding RN black hole becomes extremal. All the extrema of $r_{h}$ correspond to bifurcation points, accompanied by a change of stability of the solution.

When $\beta$ is increased from zero the basic phenomena persist and the domain $\mathcal{E}$ changes in a smooth way with some clear tendencies. The value of $r_{h}$ for which the difference between $\alpha_{\max }$ and $\alpha_{c}$ vanishes moves down quickly from its value $r_{h} \approx 0.991$ for $\beta=0$ to zero for $\beta \approx 0.757$. Hence for $\beta \geq 0.757$ the phenomenon $\alpha_{\max } \neq \alpha_{c}$ disappears. The same is true on the other limiting curve of the domain $\mathcal{E}$. The value of $\alpha$ where the extrema of $r_{h}$ merge decreases with increasing $\beta$ and becomes zero for $\beta \approx 1.18$ for the fundamental monopole (and similarly for the excited solutions). The values of $r_{h}$ with $W_{h}=0$, however, turn out to be $\beta$ independent, i.e., for $\beta>1.18$ the boundary curve of the domain $\mathcal{E}$ for $\alpha \leq \sqrt{3} / 2$ is independent of $\beta$. Fig. 6a shows the domains for some values of $\beta \leq 4$.

A new phenomenon appears for $\beta>4$, because the boundary of the allowed domain of the $\left(\alpha, r_{h}\right)$ plane for black hole boundary conditions now intersects the region $\alpha<\sqrt{3} / 2$. The curve delimiting the domain $\mathcal{E}$ towards growing $\alpha$ develops further branches as compared to $\beta \leq 4$. A picture for $\beta=6$ is shown in Fig. 6 b. Starting from the bifurcation point with the extremal RN solution at $\alpha=\sqrt{3} / 2, r_{h}=1$ the boundary curve is the straight line $\alpha=\sqrt{3} / 2$ to the intersection with the curve for an extremal horizon. Then it follows the latter curve to its extremal point $P$ with the minimal value of $\alpha$. The continuation of the curve from there with decreasing $r_{h}$ and increasing $\alpha$ is determined by a new phenomenon. The solution runs into the fixed point with $N=0$ corresponding to the conditions (20). For values of $\beta$ somewhat bigger than 4 this branch of the boundary curve is valid up to a point $Q$ with $\alpha=\sqrt{3} / 2$. The value of $r_{h}$ at $Q$ and the segment $P Q$ in Fig. 6b are drawn schematically due to lack of numerical data. From $Q$ there follows again a straight section with $\alpha=\sqrt{3} / 2$ till the curve $\alpha_{c}\left(r_{h}\right)$ already discussed for $\beta \leq 4$ branches off. The latter is then relevant down to $r_{h}=0$. With increasing $\beta$ the whole boundary curve moves inwards approaching the limiting curve for $\beta=\infty$ also shown in Fig. 6b.

\section{$7 \quad$ Stability of RN}

It is well known [18] that the Reissner-Nordstrøm black hole is a stable solution of the EinsteinMaxwell theory. Allowing for the more general perturbations provided by a non-abelian gauge field the question of stability has to be reconsidered. It was shown in 19 that the RN black holes, considered as solutions of an EYM theory, are unstable. The situation is slightly more complicated when a Higgs field is included. The Higgs field itself does not contribute unstable 
modes, moreover it has a stabilizing influence through the mass of the Yang-Mills field due to the Higgs effect. It is, in fact, easy to see that for values of the YM mass $\alpha>1 / r_{h}$ no instability of the type considered in [19] occurs. The precise value $\alpha\left(r_{h}\right)$ beyond which the RN solution becomes stable can, however, in general be determined only numerically [3, 4, except for the extremal RN black hole with $r_{h}=1$. We will show that in the latter case the solution changes stability exactly for $\alpha=\sqrt{3} / 2$. More precisely we will prove that for $\alpha<\sqrt{3} / 2$ there is an infinite number of unstable modes, all of them disappearing for $\alpha=\sqrt{3} / 2$. This is in accordance with the numerically observed bifurcation of the extremal RN black hole with the non-abelian ones (for all $n$ ) and the known change of stability at bifurcation points [20].

In order to investigate the stability we consider the linearized field equations in the $\mathrm{RN}$ background. It is easy to see [10], that the metric perturbations vanish and it suffices to study the linearized equation for $W$. With the ansatz $W=e^{i \omega t} w$ this eq. reads as

$$
-\frac{d^{2} w}{d \tau^{2}}+\left(\alpha^{2} r^{2}-1\right) w+\left(1-\frac{2}{r}\right) \frac{d w}{d \tau}=\omega^{2} \frac{r^{4}}{(r-1)^{2}} w
$$

with $r=1+e^{\tau}$. Introducing a new coordinate $\rho$ with

$$
d \rho=\frac{r^{2}}{(r-1)} d \tau
$$

Eq. (42) becomes a standard Schrödinger equation

$$
-\frac{d^{2} w}{d \rho^{2}}+V w=\omega^{2} w \quad \text { with } \quad V=\left(1-\frac{1}{r}\right)^{2}\left(\alpha^{2}-\frac{1}{r^{2}}\right) .
$$

Note that the coordinate $\rho$ tends to $-\infty$ for $r \rightarrow 1$ and to $+\infty$ for $r \rightarrow \infty$. Near the horizon $r=1$ the potential $V$ behaves like $\left(\alpha^{2}-1\right) / \rho^{2}$, hence assuming $\alpha^{2}<3 / 4$ we get $V<-1 / 4 \rho^{2}$ for $\rho \rightarrow-\infty$. According to a standard text-book theorem [21] p.1463ff, this implies the existence of infinitely many bound states for the Schrödinger eq. (44) accumulating at $\omega^{2}=0$. This proves the instability of the RN solution for $\alpha<\sqrt{3} / 2$.

For the opposite case $\alpha^{2}>3 / 4$ we shall now show that there are no bound states using the so-called Jacobi criterion. The corresponding condition is, that the zero-energy wave function $w$ with the appropriate boundary condition at $r=1$ (guaranteeing regularity) has no zero [21, 22]. Actually it suffices to study the limiting case $\alpha^{2}=3 / 4$ in view of the monotonicity of the spectrum of the Schrödinger operator with $V$.

It turns out to be convenient to use the coordinate $R=r-1$ instead of $\rho$ in this case. Putting $v=\sqrt{R} w / r$ the zero-energy Schrödinger equation reads

$$
R\left(R v^{\prime}\right)^{\prime}=\frac{3 R}{4(R+1)^{2}}\left(R^{3}+4 R^{2}+5 R-\frac{2}{3}\right) v
$$

We transform it into a Riccati equation putting $y=R v^{\prime} / v$

$$
y^{\prime}=\frac{3}{4(R+1)^{2}}\left(R^{3}+4 R^{2}+5 R-\frac{2}{3}\right)-\frac{y^{2}}{R}
$$

The shift $y \rightarrow z=y+R / 2$ leads to

$$
z^{\prime}=\frac{(R+3)^{2}}{2(R+1)^{2}}+z-\frac{z^{2}}{R}
$$

Regularity of the solution at $R=0$ requires $y(0)=0$ and hence also $z(0)=0$. From Eq. (47) it follows that $z \geq 0$ for all $R \geq 0$ and thus $y \geq-R / 2$. For large $R$ the r.h.s. of Eq. (46) is positive as long as $-R / 2 \leq y<0$ and hence $y$ is bounded from below. This shows that $v$ and thus $w$ have no zero, implying stability. 


\section{Appendix: Proof of Theorem $\mathbb{1}$}

Before entering into the proof of Theorem 1 we make some general observations concerning the behaviour of the solutions. From Eq. (11) it follows that $W(\tau)$ can have no maxima (minima) for $W>0(W<0)$ outside the ellipse $W^{2}+\alpha^{2} r^{2}=1$ in the $(r, W)$ plane. Hence a solution with $U W>0$ outside this ellipse cannot turn back to $W=0$ for increasing $\tau$.

Using the constraint Eq. (12) we may rewrite the Eq. (11b) for $N$ in the form

$$
\dot{N}=\frac{1}{2}\left(1-N^{2}-2 U^{2}-\frac{\left(W^{2}-1\right)^{2}}{r^{2}}-2 \alpha^{2} W^{2}\right),
$$

from which we see that if $N<1$ holds for some $\tau$ it remains true for all larger $\tau$. Combining Eq. (48) with the one for $\kappa$ we get

$$
(\kappa-N)^{\cdot}=-\kappa(\kappa-N)+2 U^{2}+\frac{\left(W^{2}-1\right)^{2}}{r^{2}},
$$

It follows that if $\kappa>N$ for some $\tau$ it holds for all larger $\tau$. Introducing $\eta \equiv \kappa+N-2$ we find

$$
\dot{\eta}=-\eta-\frac{1}{4} \eta^{2}-\frac{3}{4}(\kappa-N)^{2}-2 \alpha^{2} W^{2},
$$

implying $\kappa+N<2$ for large enough $\tau$.

Another useful inequality follows from the equation

$$
(1-\kappa N)^{\cdot}=-N(1-\kappa N)+2(\kappa-N) U^{2}+2 \alpha^{2} N W^{2} .
$$

Since $\kappa N \leq 1$ at the origin, resp. horizon this inequality holds for all $\tau$. Together with the constraint Eq. (12) this implies the inequality

$$
2 U^{2} \leq 1+\frac{\left(W^{2}-1\right)^{2}}{r^{2}}+2 \alpha^{2} W^{2}
$$

Hence $U$ stays bounded for $r>0$ as long as $W$ is bounded.

Let us now come to the proof of Theorem 1. It runs essentially along the same lines as the one given for $\alpha=0$ in [6], therefore we only indicate the necessary modifications of the arguments given there.

For Case i we observe that the constraint Eq. (12) implies the relation

$$
\frac{\left(W_{0}^{2}-1\right)^{2}}{r_{0}^{2}}+2 \alpha^{2} W_{0}^{2}=1+2 U_{0}^{2} \geq 1
$$

where $W_{0}, U_{0}$ and $r_{0}$ denote the values of $W, U$ and $r$ at the position of the zero of $N$. The domain of pairs $\left(r_{0}, W_{0}\right)$ allowed by this inequality is depicted as regions A and B in Fig. 2 (for $\alpha=0.75$ ). From the preceding discussion it follows that this domain is just the complement of the one allowed for black hole boundary values.

Whenever $W^{2}-1+\alpha^{2} r^{2}>0$ and $U W>0$ for some $\tau$ one necessarily ends up with Case i. The proof of this statement is completely analogous to the one for $\alpha=0$ given in Ref. [6]. In view of the assumed boundary conditions, when $\alpha \geq 1$ one obtains Case i (except for the RN solution with $W \equiv 0$ ). Therefore, in order to avoid that $N(\tau)$ develops a zero for finite $\tau$, the black hole boundary conditions have to be taken within the intersection of the domain $\mathrm{C}$ and the interior of the ellipse $\left(r_{h}, W_{h}\right): W_{h}^{2}+\alpha^{2} r_{h}^{2}=1$. This region will be denoted by $C_{+}$.

The argument showing that $r(\tau)$ turns back to $r=0$ within a finite $\tau$-interval, if $N(\tau)$ has a zero, can be taken more or less literally from [6]. 
Now let us turn to Cases ii and iii. Without restriction we may assume $\alpha \leq 1$. In these cases $N(\tau)$ stays positive and $r(\tau)$ grows monotonously, hence $r(\tau)$ is either unbounded or tends to some finite limit for $\tau \rightarrow \infty$.

In Case ii $r(\tau)$ is unbounded and we may assume that $U W<0$ for large $\tau$ (otherwise we end up with Case i). From Eqs. (11) we get, however,

$$
(U W)^{\circ}=r U^{2}+W^{2}\left(\frac{W^{2}-1}{r}+\alpha^{2} r\right)-(\kappa-N) U W,
$$

implying $(U W)^{\circ}>0$ (remember $\kappa-N>0$ ). Taking into account $W^{2} \geq 0$ and $\left(W^{2}\right)^{\circ}=r U W$, we conclude $U W \rightarrow 0$ for $\tau \rightarrow \infty$. This in turn implies $U, W \rightarrow 0$ and $\kappa, N \rightarrow 1$.

There remains only to discuss Case iii when $r(\tau)$ stays bounded. As argued in [6] this implies $N \rightarrow 0$ for $\tau \rightarrow \infty$. From the contraint (12) it then follows that the solution has to stay in the closure of region $\mathrm{A}$ in the limit as $\tau \rightarrow \infty$. Since $1-2 \alpha^{2} W^{2}$ has a positive lower bound in region $\mathrm{A}$ (except when $\alpha=1 / \sqrt{2}$ and $r=0$ ) one obtains a positive lower bound for $\kappa$ when $\tau$ is sufficiently large (see Eq. (11c)).

To prove that the solution runs into the fixpoint $U=W=0$ as claimed, we shall employ the following Ljapunov function (for the $(U, W)$ subsystem)

$$
h=\frac{1}{2} \dot{W}^{2}+\frac{1}{2} W^{2}\left(1-\alpha^{2} r^{2}-\frac{1}{2} W^{2}\right)+\delta W \dot{W} .
$$

We may consider $h(\tau)=x^{2} / 2+\left(1-\alpha^{2} r^{2}-W^{2} / 2\right) y^{2} / 2+\delta x y$ as a quadratic form in $(x, y)=$ $(\dot{W}, W)$, being positive in or close to region A for $0<\delta<\sqrt{\left(1-\alpha^{2}\right) / 2}$ and vanishing only for $\dot{W}=W=0$. The derivative of $h$ is given by

$$
\dot{h}=-\left(\left(1-\alpha^{2} r^{2}-W^{2}\right) \delta+\alpha^{2} r^{2} N\right) W^{2}-(\kappa-2 N-\delta) \dot{W}^{2}-(\kappa-2 N) \delta W \dot{W},
$$

which is a negative definite quadratic form for large $\tau$ provided $\delta$ is chosen small enough. Since two definite quadratic forms are relatively bounded there exists some constant $c>0$ such that $\dot{h} \leq-c h$ for large enough $\tau$. Hence $h(\tau) \rightarrow 0$ and thus also $U, W \rightarrow 0$ for $\tau \rightarrow \infty$. From Eqs. (11. 12) one deduces that then $\kappa$ and $r$ tend to 1 , as claimed. This concludes the proof of Theorem 1 .

\section{References}

[1] Breitenlohner, P., Forgács, P., and Maison, D.: Nucl. Phys. B 383 (1992) 357

[2] van Nieuwenhuizen, P., Wilkinson, D., and Perry, J.: Phys. Rev. D 13 (1976) 778

[3] Lee, K.-Y., Nair, V.P., and Weinberg, E J..: Phys. Rev. D 45 (1992) 2751

[4] Aichelburg, P.C., and Bizon, P.: Phys. Rev. D 48 (1993) 607

[5] Smoller, J.A., and Wasserman, A.G.: Commun. Math. Phys. 151 (1993) 303

[6] Breitenlohner, P., Forgács, P., and Maison, D.: Commun. Math. Phys. 163 (1994) 141

[7] Bartnik, R., and McKinnon, J.: Phys. Rev. Lett. 61 (1988) 141

[8] Künzle, H.P., and Masood-ul-Alam, A.K.M.: J. Math. Phys. 31 (1990) 928;

Volkov, M.S., and Galtsov, D.V.: JETP Lett. 50 (1989) 346;

Bizon, P.: Phys. Rev. Lett. 64 (1990) 2844

[9] Barriola, M., and Vilenkin, A.: Phys. Rev. Lett. 63 (1989) 341 
[10] Lee, K., Nair, V.P., and Weinberg, J.: Phys. Rev. Lett. 68 (1992) 1100

[11] Brodbeck, O., and Straumann, N.: Phys. Lett. B 324 (1994) 309

[12] Bergmann, P.G., Cahen, M., and Komar, A.B.: J. Math. Phys. 6 (1965) 1

[13] Coddington, E.A., and Levinson, N.: Theory of Ordinary Differential Equations. New York: McGraw-Hill, 1955.

[14] Hartman, P.: Ordinary Differential Equations.

Boston: Birkhäuser, 1982.

[15] Breitenlohner, P., and Maison, D.: On the Limiting Solution of the Bartnik-McKinnon Family.

Preprint MPI-PhT/94-20, May 1994, gr-qc 9405014, subm. to Commun. Math. Phys.

[16] Hollmann, H.: Phys. Lett. B 338 (1994) 181

[17] Straumann, N., and Zhou, Z.-H.: Phys. Lett. B 237 (1990) 353

[18] Moncrief, V,: Phys. Rev. D 12 (1975) 1526

[19] Bizon, P., and Wald, R.M.: Phys. Lett. B 267 (1991) 173

[20] Harrison, B.K., Thorne, K.S., Wakano M., and Wheeler, J.A.: Gravitation Theory and Gravitational Collapse.

Univ. Chicago Press, Chicago, 1965

[21] Dunford, N., and Schwartz, J.T.: Linear Operators II.

New York: Interscience Publishers, 1963

[22] Gel'fand, I.M., and Fomin, S.V.: Calculus of Variations.

Englewood Cliffs, N.J.: Prentice Hall, 1963 